\title{
Tronquée Solutions of the Third and Fourth Painlevé Equations
}

\author{
Xiaoyue XIA
}

Department of Mathematics, The Ohio State University, 100 Math Tower, 231 West 18th Avenue, Columbus OH, 43210-1174, USA

E-mail: xiaxiaoyue9@gmail.com

Received April 04, 2018, in final form August 30, 2018; Published online September 08, 2018 https://doi.org/10.3842/SIGMA.2018.095

\begin{abstract}
Recently in a paper by Lin, Dai and Tibboel, it was shown that the third and fourth Painlevé equations have tronquée and tritronquée solutions. We obtain global information about these tronquée and tritronquée solutions. We find their sectors of analyticity, their Borel summed representations in these sectors as well as the asymptotic position of the singularities near the boundaries of the analyticity sectors. We also correct slight errors in the paper mentioned.
\end{abstract}

Key words: the third and fourth Painlevé equations; asymptotic position of singularities; tronquée solutions; tritronquée solutions; Borel summed representation

2010 Mathematics Subject Classification: 34M25; 34M40; 34M55

\section{Introduction}

The well-known Painlevé equations were first introduced by Painlevé more than a century ago and have been investigated by many researchers. The Painlevé equations define new functions called Painlevé transcendents, which are considered as special nonlinear functions and their asymptotic behavior is of particular importance. For an overview of Painlevé equations and the asymptotic behavior of Painlevé transcendents please see, e.g., [2] and [14]. In recent decades there has been revived interest in Painlevé equations as they play important roles in various mathematical and physical applications (see, e.g., [2, 7, 13, 14, 22, 23] for references to applications).

Boutroux first studied a family of particular solutions of the first Painlevé equations $\mathrm{P}_{\mathrm{I}}$, which he named "tronquée" and "tritronquée" solutions in [1]. These special solutions of Painlevé equations have pole-free sectors while generic solutions have poles accumulating at $\infty$ in all sectors. Tronquée and tritronquée solutions receive attention not only for their interesting analytic property but also because they appear in a number of problems such as the Ising model [29], the critical behavior in the NLS/Toda lattices [10, 11] and the analysis of the cubic oscillator [28].

For the first Painlevé equation some pioneering works based on the powerful techniques of isomonodromic deformation and reduction to Riemann-Hilbert problem were done in the study of tronquée solutions by Kapaev and coauthors. In [20] and [24] the Stokes constant for the tritronquée solution of $\mathrm{P}_{\mathrm{I}}$ was calculated for the first time. In [22] the global asymptotic behavior of the tronquée solutions of $\mathrm{P}_{\mathrm{I}}$ was described with connection formulae presented. In [23] and [17] the global asymptotic behavior of the tronquée solutions of $\mathrm{P}_{\text {II }}$ was described with connection formulae presented. In [21] the global asymptotics of the solutions of the fourth

This paper is a contribution to the Special Issue on Painlevé Equations and Applications in Memory of Andrei Kapaev. The full collection is available at https://www.emis.de/journals/SIGMA/Kapaev.html 
Painlevé equation $\mathrm{P}_{\mathrm{IV}}$ including its tronquée solutions was analyzed in detail. In [12] an fourthorder nonlinear ODE which controls the pole dynamics in the general solution of equation $\mathrm{P}_{\mathrm{I}}^{2}$ was studied. See also the monograph [14] for a summary of recent developments in the theory of Painlevé equations based on this Riemann-Hilbert-isomonodromy method.

There is an impressive body of work on tronquée solutions and we only mention a few contributions here. Using approaches different from the Riemann-Hilbert-isomonodromy method Costin and coauthors analyzed tronquée solutions of $\mathrm{P}_{\mathrm{I}}$ in [9] and [8] and obtained similar results to those in [20] and [24]. In [15] the existence of the tritronquée solutions of $\mathrm{P}_{\mathrm{I}}^{2}$, the second member in the $\mathrm{P}_{\mathrm{I}}$ hierarchy was proved. In [19] the existence of tronquée solutions of the second Painlevé hierarchy was proved. For the location of poles for the Hasting-McLeod solution to the second Painlevé equation please see [16], in which a special case of Novokshenov conjecture [31] was also proved. For the tronquée solutions to the third Painlevé equation please see [27], which followed the idea in [18].

In this paper the tronquée and tritronquée solutions of the third and fourth Painlevé equation are studied:

$$
\begin{aligned}
& \mathrm{P}_{\mathrm{III}}: \frac{\mathrm{d}^{2} y}{\mathrm{~d} x^{2}}=\frac{1}{y}\left(\frac{\mathrm{d} y}{\mathrm{~d} x}\right)^{2}-\frac{1}{x} \frac{\mathrm{d} y}{\mathrm{~d} x}+\frac{1}{x}\left(\alpha y^{2}+\beta\right)+\gamma y^{3}+\frac{\delta}{y}, \\
& \mathrm{P}_{\mathrm{IV}}: \frac{\mathrm{d}^{2} y}{\mathrm{~d} x^{2}}=\frac{1}{2 y}\left(\frac{\mathrm{d} y}{\mathrm{~d} x}\right)^{2}+\frac{3}{2} y^{3}+4 x y^{2}+2\left(x^{2}-\alpha\right) y+\frac{\beta}{y},
\end{aligned}
$$

where $\alpha, \beta, \gamma$ and $\delta$ are arbitrary complex numbers. By Bäcklund transformations (see [30]) $\mathrm{P}_{\text {III }}$ can be reduced to

$$
\begin{aligned}
& \mathrm{P}_{\mathrm{III}}^{(i)}: \frac{\mathrm{d}^{2} y}{\mathrm{~d} x^{2}}=\frac{1}{y}\left(\frac{\mathrm{d} y}{\mathrm{~d} x}\right)^{2}-\frac{1}{x} \frac{\mathrm{d} y}{\mathrm{~d} x}+\frac{1}{x}\left(\alpha y^{2}+\beta\right)+y^{3}-\frac{1}{y}, \\
& \mathrm{P}_{\mathrm{III}}^{(i i)}: \frac{\mathrm{d}^{2} y}{\mathrm{~d} x^{2}}=\frac{1}{y}\left(\frac{\mathrm{d} y}{\mathrm{~d} x}\right)^{2}-\frac{1}{x} \frac{\mathrm{d} y}{\mathrm{~d} x}+\frac{1}{x}\left(y^{2}+\beta\right)-\frac{1}{y} .
\end{aligned}
$$

In a famous paper [29] by McCoy, Tracy and $\mathrm{Wu}$, a one-parameter family of tronquée solutions of a special case of (1.2) where $\alpha=2 \nu, \beta=-2 \nu$ was constructed, whose asymptotics at $\infty$ was congruent to ours $((2.1)$ and (3.1)) and asymptotic expansion for small $x$ was obtained. Furthermore, in a recent paper [13] by Fasondini et al. a comprehensive computer simulation of the McCoy-Tracy-Wu solution was given. The computer pictures of the pole distributions in [13] provide a good illustration of our description of the asymptotic position of poles in, e.g., (2.16).

(1.3) was studied as the degenerate $\mathrm{P}_{\mathrm{III}}$ in [25] and [26], and the position of the first array of poles was found in [26] via isomonodromy methods.

We base our methods on the results in [3] and [6], which used the technique of Borel summation to describe the Stokes phenomenon. We obtain representations of tronquée solutions as Borel summed transseries (see also [5]), as well as the position of the first array of poles, bordering the sector of analyticity. We will first use a simple example to briefly illustrate some concepts in the Borel summation method. Please see also [8, Section 5] for an introduction.

In the following, we denote by $\mathcal{L}_{\phi}$ the Laplace transform

$$
f \longmapsto \int_{0}^{\infty \mathrm{e}^{\mathrm{i} \phi}} f(p) \mathrm{e}^{-x p} \mathrm{~d} p,
$$

where $\phi \in \mathbb{R}$. See also $[3$, p. 8] for the notation.

Assume that we have a formal series

$$
\tilde{f}(w)=\sum_{n=0}^{\infty} a_{n} w^{-r-n}, \quad \operatorname{Re}(r)>0,
$$


where the series $\sum_{n=0}^{\infty} a_{n} x^{n}$ has a positive radius of convergence. The Borel transform of $\tilde{f}$ is defined to be the formal power series

$$
(\mathcal{B} \tilde{f})(p):=\sum_{n=0}^{\infty} \frac{a_{n} p^{n+r-1}}{\Gamma(n+r)} .
$$

In most cases the explicit solution of a differential equation is not known. We may obtain classical asymptotic series as formal power series solutions, but these formal solutions do not contain parameters that help us distinguish between actual solutions. This is illustrated in the following simple ordinary differential equation at the irregular singularity at $x=\infty$

$$
y^{\prime}+y=\frac{1}{x^{2}}
$$

The unique formal power series solution for $x \rightarrow \infty$ is

$$
\tilde{y}_{0}(x)=\sum_{n=1}^{\infty} \frac{n !}{x^{n+1}}
$$

and the general solution to (1.4) is

$$
y(x ; C)=y_{0}(x)+C \mathrm{e}^{-x}, \quad \text { where } \quad y_{0}(x)=\mathrm{e}^{-x} \int_{x_{1}}^{x} \frac{\mathrm{e}^{s}}{s^{2}} \mathrm{~d} s \sim \tilde{y}_{0}(x) \quad \text { as } \quad x \rightarrow+\infty .
$$

The idea of transseries solution is a completion of classical formal power series solution in the sense that the transseries solution representation includes the free parameters which appear in the actual solutions. In the example above, if we let

$$
\tilde{y}(x)=\tilde{y}_{0}(x)+C \mathrm{e}^{-x} \quad \text { for } \quad x \rightarrow+\infty,
$$

then (1.5) is a formal solution to (1.4) and the simplest example of a transseries.

Under appropriate conditions (see $[3,4,6]$ ), given $\phi$, the operator $\mathcal{L}_{\phi} \mathcal{B}$ is a one-to-one map between the transseries solutions and actual solutions. In the example (1.4), the actual solutions have representation:

$$
y(x)=\left\{\begin{array}{l}
\mathcal{L}_{\phi} \mathcal{B} \tilde{y}_{0}(x)+C_{+} \mathrm{e}^{-x}, \quad-\phi=\arg (x) \in\left(0, \frac{\pi}{2}\right), \\
\mathcal{L}_{\phi} \mathcal{B} \tilde{y}_{0}(x)+C_{-} \mathrm{e}^{-x}, \quad-\phi=\arg (x) \in\left(-\frac{\pi}{2}, 0\right) .
\end{array}\right.
$$

The value $C_{+}-C_{-}$is called the Stokes constant. This representation is a trivial example of Borel summed representation of solutions. In the case of nonlinear systems such as (1.8), the transseries solution is of the form (4.1) and the Borel summed representation of actual solutions is of the form (4.3).

In this paper we study tronquée solutions of (1.2), (1.3) and (1.1) by first transforming each of them into a second-order differential equation of the following form

$$
h^{\prime \prime}(w)-h(w)+\frac{1}{w}\left[\left(\beta_{2}-\beta_{1}\right) h(w)+\left(\beta_{2}+\beta_{1}\right) h^{\prime}(w)\right]=g\left(w, h, h^{\prime}\right),
$$

where $\beta_{1}$ and $\beta_{2}$ are constants and $g\left(w, h, h^{\prime}\right)$ is analytic at $(\infty, 0,0)$. See (3.1) for the change of variable for (1.2), see (3.7) for the change of variable for (1.3) and see (3.12), (3.18) and (3.25) for the change of variable for (1.1). Next we make the substitution

$$
\left[\begin{array}{l}
h(w) \\
h^{\prime}(w)
\end{array}\right]=\left[\begin{array}{rr}
1-\frac{\beta_{1}}{2 w} & 1+\frac{\beta_{2}}{2 w} \\
-1-\frac{\beta_{1}}{2 w} & 1-\frac{\beta_{2}}{2 w}
\end{array}\right] \mathbf{u}(w) .
$$


Then $\mathbf{u}$ is a solution to the following normalized (see [6]) 2-dimensional differential system:

$$
\mathbf{u}^{\prime}+\left(\hat{\Lambda}+\frac{\hat{B}}{w}\right) \mathbf{u}=\mathbf{g}(w, \mathbf{u})
$$

where

$$
\hat{\Lambda}=\left[\begin{array}{cc}
1 & 0 \\
0 & -1
\end{array}\right], \quad \hat{B}=\left[\begin{array}{cc}
\beta_{1} & 0 \\
0 & \beta_{2}
\end{array}\right],
$$

and $\mathbf{g}(w, \mathbf{u})$ is analytic at $(\infty, \mathbf{0})$ with $\mathbf{g}(w, \mathbf{u})=O\left(w^{-2}\right)+O\left(|\mathbf{u}|^{2}\right)$ as $w \rightarrow \infty$ and $\mathbf{u} \rightarrow \mathbf{0}$.

We obtain information about the tronquée and tritronquée solutions of the normalized system (1.8) such as their existence, regions of analyticity and asymptotic position of poles through which we obtain corresponding results regarding tronquée and tritronquée solutions of $\mathrm{P}_{\mathrm{III}}$ and $\mathrm{P}_{\mathrm{IV}}$. See also [8] in which a similar approach was used to study the tronquée solutions of the first Painlevé equation.

\section{Tronquée solutions of (1.6)}

\subsection{Formal solutions and tronquée solutions of (1.6)}

In Proposition 2.1 and Theorem 2.2 we present formal and actual solutions on the right half $w$-plane $S_{1}:=\left\{w: \arg (w) \in\left(-\frac{\pi}{2}, \frac{\pi}{2}\right)\right\}$. Then through a simple symmetry transformation we obtain solutions in the left half plane $S_{2}:=\left\{w: \arg (w) \in\left(\frac{\pi}{2}, \frac{3 \pi}{2}\right)\right\}$. We start with the formal expansions of the solutions.

Assume that $d$ is a ray of the form $\mathrm{e}^{\mathrm{i} \phi} \mathbb{R}^{+}$with $\phi \in\left(-\frac{\pi}{2}, \frac{\pi}{2}\right)$. We have the following results on transseries solutions, formal expansions in powers of $1 / w$ and $\mathrm{e}^{-w}$ (see [6]), of (1.6) valid on $d$ and, moreover, in the sector $S_{1}$ :

Proposition 2.1. Assume that $d$ is a ray of the form $\mathrm{e}^{\mathrm{i} \phi} \mathbb{R}^{+}$with $\phi \in\left(-\frac{\pi}{2}, \frac{\pi}{2}\right)$. Then

(i) the one-parameter family of transseries solutions of (1.6) satisfying $h(w) \rightarrow 0$ as $|w| \rightarrow \infty$ on $d$ are

$$
\tilde{h}(w)=\tilde{h}_{0}(w)+\sum_{k=1}^{\infty} C^{k} \mathrm{e}^{-k w} w^{-\beta_{1} k} \tilde{s}_{k}(w),
$$

where for each $k \geq 1$

$$
\tilde{s}_{k}(w)=\sum_{j=0}^{\infty} \frac{s_{k, j}}{w^{j}}
$$

is a formal power series in $w^{-1}$.

(ii) The formal power series in $w^{-1}$

$$
\tilde{h}_{0}(w)=\sum_{j=2}^{\infty} \frac{h_{0, j}}{w^{j}}
$$

is the unique formal power series solution of (1.6). 
The results in [6] provide us with the relation between these transseries solutions and actual solutions.

In the following, we denote by $\mathcal{L}_{\phi}$ the Laplace transform

$$
f \longmapsto \int_{0}^{\infty \mathrm{e}^{\mathrm{i} \phi}} f(p) \mathrm{e}^{-x p} \mathrm{~d} p,
$$

where $\phi \in \mathbb{R}$. See also [3, p. 8] for the notation.

Theorem 2.2. Let $d, \tilde{h}_{0}(w)$ and $\tilde{s}_{k}(w)$ be as in Proposition 2.1. Let $h(w)$ be a solution to (1.6) on $d$ for $|w|$ large enough satisfying

$$
h(w) \rightarrow 0, \quad w \in d, \quad|w| \rightarrow \infty .
$$

Then

(i) There is a unique pair of constants $\left(C_{+}, C_{-}\right)$associated with $h(w)$, and $h(w)$ has the following representations

$$
\begin{aligned}
& h(w)=\mathcal{L}_{\phi} H_{0}(w)+\sum_{k=1}^{\infty} C_{+}^{k} \mathrm{e}^{-k w} w^{k M_{1}} \mathcal{L}_{\phi} H_{k}(w), \quad-\phi=\arg (w) \in\left(0, \frac{\pi}{2}\right), \\
& h(w)=\mathcal{L}_{\phi} H_{0}(w)+\sum_{k=1}^{\infty} C_{-}^{k} \mathrm{e}^{-k w} w^{k M_{1}} \mathcal{L}_{\phi} H_{k}(w), \quad-\phi=\arg (w) \in\left(-\frac{\pi}{2}, 0\right),
\end{aligned}
$$

where

$$
\begin{aligned}
& M_{1}=\left\lfloor\operatorname{Re}\left(-\beta_{1}\right)\right\rfloor+1, \quad H_{0}=\mathcal{B} \tilde{h}_{0}, \\
& H_{k}=\mathcal{B} \tilde{h}_{k}=\mathcal{B}\left(w^{-k \beta_{1}-k M_{1}} \tilde{s}_{k}\right), \quad k=1,2, \ldots,
\end{aligned}
$$

where each $H_{k}$ is analytic on the Riemann surface of $\mathbb{C} \backslash\left(\mathbb{Z}^{+} \cup \mathbb{Z}^{-}\right)$, and the branch cut for each $H_{k}, k \geq 1$, is chosen to be $(-\infty, 0]$.

(ii) There exists $\epsilon_{0}>0$ such that for each $0<\epsilon \leq \epsilon_{0}$ there exist $\delta_{\epsilon}>0, R_{\epsilon}>0$ such that $h(w)$ can be analytically continued to (at least) the following region

$$
S_{\mathrm{an}, \epsilon}(h(w))=S_{\epsilon}^{+} \cup S_{\epsilon}^{-},
$$

where

$$
\begin{aligned}
& S_{\epsilon}^{-}=\left\{w:|w|>R_{\epsilon}, \arg (w) \in\left[-\frac{\pi}{2}-\epsilon, \frac{\pi}{2}-\epsilon\right] \text { and }\left|C_{-} \mathrm{e}^{-w} w^{-\beta_{1}}\right|<\delta_{\epsilon}^{-1}\right\}, \\
& S_{\epsilon}^{+}=\left\{w:|w|>R_{\epsilon}, \arg (w) \in\left[-\frac{\pi}{2}+\epsilon, \frac{\pi}{2}+\epsilon\right] \text { and }\left|C_{+} \mathrm{e}^{-w} w^{-\beta_{1}}\right|<\delta_{\epsilon}^{-1}\right\} .
\end{aligned}
$$

Consequently, $h(w)$ is analytic (at least) in

$$
S_{\mathrm{an}}(h)=\bigcup_{0<\epsilon \leq \epsilon_{0}}\left(S_{\epsilon}^{-} \cup S_{\epsilon}^{+}\right) .
$$

(iii) $h(w) \sim h_{0}(w)$ in $S_{1}$.

\section{Note 2.3.}

(i) It is straightforward to check that if $\operatorname{Re}\left(\beta_{1}\right)>0, S_{\text {an }}$ contains all but a compact subset of i $\mathbb{R}$. In other words there exists $R_{0}>0$ such that $h(w)$ is analytic in the closure of $S_{1} \backslash \mathbb{D}_{R_{0}}$, where $S_{1}$ is the open right half plane and $\mathbb{D}_{R_{0}}=\left\{|w|<R_{0}\right\}$ is the open disk centered at origin with radius $R_{0}$. 
(ii) On the other hand if $\operatorname{Re}\left(\beta_{1}\right)<0, S_{\text {an }}^{c}$ contains all but a compact subset of iR. We point out that in particular, the solution is not analytic in $S_{1} \backslash \mathbb{D}_{R_{0}}$ for any $R_{0}>0$, contrary to the claim in [27]. Singularities of the tronquée solutions exist for large $w$ in $S_{1}$ as seen in Theorem 2.4, 2.5, 3.2, 3.3, 3.9 and 3.10.

Theorem 2.4 (asymptotic position of singularities). Let $h, C_{+}$and $C_{-}$be as in Theorem 2.2.

(i) Assume $C_{+} \neq 0$. Denote

$$
\xi_{+}(w)=C_{+} w^{-\beta_{1}} \mathrm{e}^{-w} .
$$

Then

$$
h(w) \sim \sum_{m=0}^{\infty} \frac{F_{m}\left(\xi_{+}(w)\right)}{w^{m}}, \quad|w| \rightarrow \infty, \quad w \in \mathcal{D}_{w}^{+},
$$

where for each $m \geq 0, F_{m}$ is analytic at $\xi=0$ and

$$
\mathcal{D}_{w}^{+}=\left\{|w|>R: \arg w \in\left(-\frac{\pi}{2}+\delta, \frac{\pi}{2}+\delta\right), \operatorname{dist}\left(\xi_{+}(w), \Xi\right)>\epsilon,|\xi(w)|<\epsilon^{-1}\right\}
$$

for any $\delta, \epsilon>0$ small enough and $R$ large enough, and where $\Xi$ is the set of singularities of $F_{0}(\xi) . F_{0}(\xi)$ satisfies

$$
F_{0}(0)=0, \quad F_{0}^{\prime}(0)=1 .
$$

(ii) Assume $C_{+} \neq 0$, and $\xi_{s} \in \Xi$ is a singularity of $F_{0}$. Then the singular points of $h, w_{n}^{+}$, near the boundary $\{w: \arg (w)=\pi / 2\}$ of the sector of analyticity are given asymptotically by

$$
w_{n}^{+}=2 n \pi \mathrm{i}-\beta_{1} \ln (2 n \pi \mathrm{i})+\ln \left(C_{+}\right)-\ln \left(\xi_{s}\right)+o(1)
$$

as $n \rightarrow \infty$.

(iii) Assume $C_{-} \neq 0$. Denote

$$
\xi_{-}(w)=C_{-} w^{-\beta_{1}} \mathrm{e}^{-w} .
$$

Then

$$
h(w) \sim \sum_{m=0}^{\infty} \frac{F_{m}\left(\xi_{-}(w)\right)}{w^{m}}, \quad|w| \rightarrow \infty, \quad w \in \mathcal{D}_{w}^{-},
$$

where

$$
\mathcal{D}_{w}^{-}=\left\{|w|>R: \arg w \in\left(-\frac{\pi}{2}-\delta, \frac{\pi}{2}-\delta\right), \operatorname{dist}\left(\xi_{-}(w), \Xi\right)>\epsilon,|\xi(w)|<\epsilon^{-1}\right\}
$$

for any $\delta, \epsilon>0$ small enough and $R$ large enough, and where $F_{m}, m \geq 0$, and $\Xi$ are as described in $(i)$.

(iv) Assume $C_{-} \neq 0$, and $\xi_{s} \in \Xi$ is a singularity of $F_{0}$. Then the singular points of $h, w_{n}^{-}$, near the boundary $\{w: \arg (w)=-\pi / 2\}$ of the sector of analyticity are given asymptotically by

$$
w_{n}^{-}=-2 n \pi \mathrm{i}-\beta_{1} \ln (-2 n \pi \mathrm{i})+\ln \left(C_{-}\right)-\ln \left(\xi_{s}\right)+o(1)
$$

as $n \rightarrow \infty$.

The expression of $F_{0}$ (see (3.5), (3.11), (3.17) and (3.23)) is obtained explicitly in each case where asymptotic position of singularities is presented. 


\subsection{Tritronquée solutions of (1.6)}

The information on formal and actual tronquée solutions of (1.6) in the left half plane $S_{2}:=$ $\left\{w: \arg (w) \in\left(\frac{\pi}{2}, \frac{3 \pi}{2}\right)\right\}$ is obtained by means of a simple transformation

$$
h(w)=\hat{h}(-w), \quad \tilde{w}=-w .
$$

(1.6) is rewritten as

$$
\hat{h}^{\prime \prime}(\tilde{w})-\hat{h}(\tilde{w})+\frac{1}{\tilde{w}}\left[\left(\beta_{1}-\beta_{2}\right) \hat{h}(\tilde{w})+\left(\beta_{1}+\beta_{2}\right) \hat{h}^{\prime}(\tilde{w})\right]=g\left(-\tilde{w}, \hat{h},-\hat{h}^{\prime}\right),
$$

which is of the form (1.6) with $\beta_{1}$ and $\beta_{2}$ exchanged, and thus all results in Proposition 2.1, Theorems 2.2 and 2.4 apply. Without repeating all of the results, we introduce some notations needed for describing the tritronquée solutions of (1.6).

The small transseries solutions of (2.13) in the right half $\tilde{w}$-plane is

$$
\tilde{h}_{l}(\tilde{w})=\tilde{h}_{0}(-\tilde{w})+\sum_{k=1}^{\infty} C^{k} \mathrm{e}^{-k \tilde{w}} \tilde{w}^{-\beta_{2} k} \tilde{t}_{k}(\tilde{w}),
$$

where for each $k \geq 1, \tilde{t}_{k}(\tilde{w})$ is a formal power series in $\tilde{w}^{-1}$.

Assume that $\hat{h}(\tilde{w})$ is an actual solution to $(2.13)$ on $d=\mathrm{e}^{\mathrm{i} \theta} \mathbb{R}^{+}$with $\cos \theta>0$, such that $\hat{h}(\tilde{w})=o(1)$ as $|\tilde{w}| \rightarrow \infty$. Then there exists a unique pair of constants $\left(\hat{C}_{+}, \hat{C}_{-}\right)$such that

$$
\hat{h}(\tilde{w})=\left\{\begin{array}{l}
\mathcal{L}_{\phi} \hat{H}_{0}(\tilde{w})+\sum_{k=1}^{\infty} \hat{C}_{+}^{k} \mathrm{e}^{-k \tilde{w}} \tilde{w}^{k M_{2}} \mathcal{L}_{\phi} \hat{H}_{k}(\tilde{w}), \quad-\phi=\arg (\tilde{w}) \in\left(0, \frac{\pi}{2}\right), \\
\mathcal{L}_{\phi} \hat{H}_{0}(\tilde{w})+\sum_{k=1}^{\infty} \hat{C}_{-}^{k} \mathrm{e}^{-k \tilde{w}} \tilde{w}^{k M_{2}} \mathcal{L}_{\phi} \hat{H}_{k}(\tilde{w}), \quad-\phi=\arg (\tilde{w}) \in\left(-\frac{\pi}{2}, 0\right),
\end{array}\right.
$$

where

$$
M_{2}=\left\lfloor\operatorname{Re}\left(-\beta_{2}\right)\right\rfloor+1, \quad \hat{H}_{0}(p)=-H_{0}(-p), \quad \hat{H}_{k}=\mathcal{B}\left(w^{-k \beta_{2}-k M_{2}} \tilde{t}_{k}\right), \quad k \geq 1
$$

where each $\hat{H}_{k}$ is analytic in the Riemann surface of $\mathbb{C} \backslash\left(\mathbb{Z}^{+} \cup \mathbb{Z}^{-}\right)$, and the branch cut for each $\hat{H}_{k}, k \geq 1$, is chosen to be $(-\infty, 0]$. Note that the second equation in (2.14) holds because the power series solution of $(2.13)$ must be $\tilde{h}_{0}(-\tilde{w})$. By the definition of the Borel transform (see Appendix A) we have $\hat{H}_{0}(p)=-H_{0}(-p)$.

By Theorem 2.2(ii), $\hat{h}$ is analytic at least on

$$
\hat{S}_{\mathrm{an}}(h):=-S_{\mathrm{an}}(\hat{h}),
$$

where $S_{\text {an }}(\hat{h})$ is given by $(2.4)-(2.6)$ with $\beta_{1}$ replaced by $\beta_{2}$. Denote $\hat{\xi}_{ \pm}=\hat{C}_{ \pm} \tilde{w}^{-\beta_{2}} \mathrm{e}^{-\tilde{w}}$ as in $(2.7)$ and (2.11). By Theorem 2.4 if $\hat{C}_{+} \neq 0$ then

$$
\hat{h}(\tilde{w}) \sim \sum_{m=0}^{\infty} \frac{\hat{F}_{m}\left(\hat{\xi}_{+}(\tilde{w})\right)}{\tilde{w}^{m}}, \quad|\tilde{w}| \rightarrow \infty, \quad \tilde{w} \in \mathcal{D}_{\tilde{w}}^{+},
$$

where $\hat{F}_{m}$ are analytic at $\xi=0$. If $\hat{C}_{-} \neq 0$ then

$$
\hat{h}(\tilde{w}) \sim \sum_{m=0}^{\infty} \frac{\hat{F}_{m}\left(\hat{\xi}_{-}(\tilde{w})\right)}{\tilde{w}^{m}}, \quad|\tilde{w}| \rightarrow \infty, \quad \tilde{w} \in \mathcal{D}_{\tilde{w}}^{-},
$$


where $D_{\tilde{w}}^{ \pm}$are defined by $(2.8)$ and $(2.12)$ with $\xi_{ \pm}$replaced by $\hat{\xi}_{ \pm}$respectively and $\Xi$ replaced by $\hat{\Xi}$ which is defined to be the set of singularities of $\hat{F}_{0}$.

Tritronquée solutions are special cases of tronquée solutions with $C_{+}=0$ or $C_{-}=0$. Denote

$$
\begin{aligned}
& h^{+}(w)=\mathcal{L}_{\phi} H_{0}(w), \quad-\phi=\arg (w) \in(0, \pi), \\
& h^{-}(w)=\mathcal{L}_{\phi} H_{0}(w), \quad-\phi=\arg (w) \in(-\pi, 0), \\
& \hat{h}^{+}(w)=\mathcal{L}_{\phi} \hat{H}_{0}(w), \quad-\phi=\arg (w) \in(0, \pi), \\
& \hat{h}^{-}(w)=\mathcal{L}_{\phi} \hat{H}_{0}(w), \quad-\phi=\arg (w) \in(-\pi, 0) \text {. }
\end{aligned}
$$

Corollary 2.5. Assume $\phi \in\left(0, \frac{\pi}{2}\right)$. Let $C_{1}^{t}, C_{2}^{t}, C_{3}^{t}, C_{4}^{t}$ be the constants in the transseries of $h^{ \pm}$and $\hat{h}^{ \pm}$, namely,

$$
\begin{aligned}
& h^{+}(w)=\mathcal{L}_{-\phi} H_{0}(w)=\mathcal{L}_{\phi} H_{0}(w)+\sum_{k=1}^{\infty}\left(C_{1}^{t}\right)^{k} \mathrm{e}^{-k w} w^{M_{1} k} \mathcal{L}_{\phi} H_{k}(w), \\
& h^{-}(w)=\mathcal{L}_{\phi} H_{0}(w)=\mathcal{L}_{-\phi} H_{0}(w)+\sum_{k=1}^{\infty}\left(C_{2}^{t}\right)^{k} \mathrm{e}^{-k w} w^{M_{1} k} \mathcal{L}_{-\phi} H_{k}(w), \\
& \hat{h}^{+}(w)=\mathcal{L}_{-\phi} \hat{H}_{0}(w)=\mathcal{L}_{\phi} \hat{H}_{0}(w)+\sum_{k=1}^{\infty}\left(C_{3}^{t}\right)^{k} \mathrm{e}^{-k w} w^{M_{2} k} \mathcal{L}_{\phi} \hat{H}_{k}(w), \\
& \hat{h}^{-}(w)=\mathcal{L}_{\phi} \hat{H}_{0}(w)=\mathcal{L}_{-\phi} \hat{H}_{0}(w)+\sum_{k=1}^{\infty}\left(C_{4}^{t}\right)^{k} \mathrm{e}^{-k w} w^{M_{2} k} \mathcal{L}_{-\phi} \hat{H}_{k}(w) .
\end{aligned}
$$

(i) We have

$$
h^{+}(w)=\hat{h}^{-}(-w), \quad h^{-}(w)=\hat{h}^{+}(-w) .
$$

A consequence of Theorem 2.2(ii) is that for any $\delta>0$ there exists $R>0$ such that $h^{+}$is analytic in the sector

$$
T_{\delta, R}^{+}:=\left\{w:|w|>R, \arg (w) \in\left[-\frac{\pi}{2}+\delta, \frac{3 \pi}{2}-\delta\right]\right\}
$$

and $h^{-}$is analytic in the sector

$$
T_{\delta, R}^{-}:=\left\{w:|w|>R, \arg (w) \in\left[-\frac{3 \pi}{2}+\delta, \frac{\pi}{2}-\delta\right]\right\}
$$

(ii) Assume $\xi_{s} \in \Xi$ is a singularity of $F_{0}$ (see Theorem $2.4(i i)$ ) and $\hat{\xi}_{s} \in \hat{\Xi}$ is a singularity of $\hat{F}_{0}$. Then the singular points of $h^{+}, w_{1, n}^{-}$near the boundary $\left\{w: \arg w=-\frac{\pi}{2}\right\}$ and $w_{1, n}^{+}$ near the boundary $\left\{w: \arg w=\frac{3 \pi}{2}\right\}$, are given asymptotically by

$$
\begin{aligned}
& w_{1, n}^{-}=-2 n \pi \mathrm{i}-\beta_{1} \ln (-2 n \pi \mathrm{i})+\ln \left(C_{1}^{t}\right)-\ln \left(\xi_{s}\right)+o(1), \\
& w_{1, n}^{+}=-2 n \pi \mathrm{i}+\beta_{2} \ln (2 n \pi \mathrm{i})-\ln \left(C_{4}^{t}\right)+\ln \left(\hat{\xi}_{s}\right)+o(1),
\end{aligned}
$$

as $n \rightarrow \infty$. The singular points of $h^{-}, w_{2, n}^{-}$near the boundary $\left\{w: \arg w=-\frac{3 \pi}{2}\right\}$ and $w_{2, n}^{+}$ near the boundary $\left\{w: \arg w=\frac{\pi}{2}\right\}$, are given asymptotically by

$$
\begin{aligned}
& w_{2, n}^{-}=2 n \pi \mathrm{i}+\beta_{2} \ln (-2 n \pi \mathrm{i})-\ln \left(C_{3}^{t}\right)+\ln \left(\hat{\xi}_{s}\right)+o(1), \\
& w_{2, n}^{+}=2 n \pi \mathrm{i}-\beta_{1} \ln (2 n \pi \mathrm{i})+\ln \left(C_{2}^{t}\right)-\ln \left(\xi_{s}\right)+o(1) .
\end{aligned}
$$




\section{Normalizations and Tronquée solutions of $\mathbf{P}_{\mathrm{III}}$ and $\mathbf{P}_{\mathrm{IV}}$}

\subsection{Tronquée solutions of $\mathbf{P}_{\text {III }}^{(i)}$}

If $y(x)$ is a solution of (1.2) which is asymptotic to a formal power series on a ray $d$ which is not an antistokes line (lines on which $\arg w= \pm \frac{\pi}{2}$ where $w$ is the independent variable in the normalized equation), then by dominant balance we have

$$
y(x) \sim l(x), \quad|x| \rightarrow \infty, \quad x \in d,
$$

where

$$
l(x)=A-\left(\frac{\alpha+A^{2} \beta}{4}\right) \frac{1}{x}
$$

for some $A$ satisfying $A^{4}=1$. Fix some $A$ satisfying $A^{4}=1$ and make the change of variables

$$
w=2 A x, \quad y(x)=h(w)+l\left(\frac{w}{2 A}\right) .
$$

Then the equation (1.2) is transformed into an equation for $h$ of the form (1.6) with

$$
\beta_{1}=\frac{1}{2}+\frac{\alpha}{4}-\frac{A^{2} \beta}{4}, \quad \beta_{2}=\frac{1}{2}-\frac{\alpha}{4}+\frac{A^{2} \beta}{4} .
$$

Results in Section 2 apply. Let the notations be the same as in Section 2.

\section{Theorem 3.1.}

(i) There is a unique formal power series solution

$$
\tilde{y}_{0}(x)=\sum_{k=0}^{\infty} \frac{y_{0, k}}{x^{k}}
$$

to (1.2), where

$$
y_{0,0}=A, \quad y_{0,1}=-\frac{\alpha+A^{2} \beta}{4} .
$$

(ii) There is a one-parameter family $\mathcal{F}_{A, 1}$ of tronquée solutions of (1.2) in $A^{-1} S_{1}$ with representations

$$
y(x)=\left\{\begin{array}{l}
l(x)+h^{+}(2 A x)+\sum_{k=1}^{\infty} C_{+}^{k} \mathrm{e}^{-2 A k x}(2 A x)^{k M_{1}} \mathcal{L}_{\phi} H_{k}(2 A x), \quad-\phi \in\left(0, \frac{\pi}{2}\right), \\
l(x)+h^{-}(2 A x)+\sum_{k=1}^{\infty} C_{-}^{k} \mathrm{e}^{-2 A k x}(2 A x)^{k M_{1}} \mathcal{L}_{\phi} H_{k}(2 A x), \quad-\phi \in\left(-\frac{\pi}{2}, 0\right) .
\end{array}\right.
$$

(iii) There is a one-parameter family $\mathcal{F}_{A, 2}$ of tronquée solutions of (1.2) in $A^{-1} S_{2}$ with representations

$$
y(x)= \begin{cases}l(x)+h^{-}(2 A x)+\sum_{k=1}^{\infty} \hat{C}_{+}^{k} \mathrm{e}^{2 A k x}(-2 A x)^{k M_{2}} \mathcal{L}_{\phi} \hat{H}_{k}(-2 A x), & -\phi \in\left(0, \frac{\pi}{2}\right), \\ l(x)+h^{+}(2 A x)+\sum_{k=1}^{\infty} \hat{C}_{-}^{k} \mathrm{e}^{2 A k x}(-2 A x)^{k M_{2}} \mathcal{L}_{\phi} \hat{H}_{k}(-2 A x), & -\phi \in\left(-\frac{\pi}{2}, 0\right) .\end{cases}
$$


(iv) For each tronquée solution in (ii) or (iii) we have

$$
y(x) \sim \tilde{y}_{0}(x), \quad x \in d=A^{-1} \mathrm{e}^{\mathrm{i} \theta} \mathbb{R}^{+}, \quad|x| \rightarrow \infty,
$$

and the solution is analytic at least in $(2 A)^{-1} S_{\text {an }}$ if $\cos \theta>0$, in $(2 A)^{-1} \hat{S}_{\text {an }}$ if $\cos \theta<0$. $S_{\text {an }}$ and $\hat{S}_{\text {an }}$ are as defined in Theorem 2.2 and Section 2.2 .

From Theorem 2.4 we obtain information about the singularities of $y$. Assume that $y$ is a tronquée solution with representation (3.2) or (3.3). Let $\xi_{+}=C_{+} \mathrm{e}^{-w} w^{-\beta_{1}}, \xi_{-}=C_{-} \mathrm{e}^{-w} w^{-\beta_{2}}$, $F_{m}$ and $\hat{F}_{m}$ be as in Section 2. Then the equation satisfied by $F_{0}$ is

$$
\xi^{2} \frac{\mathrm{d}^{2}}{\mathrm{~d} \xi^{2}} F_{0}(\xi)+\xi \frac{\mathrm{d}}{\mathrm{d} \xi} F_{0}(\xi)-\frac{\xi^{2}\left(\frac{\mathrm{d}}{\mathrm{d} \xi} F_{0}(\xi)\right)^{2}}{A+F_{0}(\xi)}-\frac{\left(A+F_{0}(\xi)\right)^{3}}{4 A^{2}}+\frac{1}{4 A^{2}\left(A+F_{0}(\xi)\right)}=0 .
$$

The equation of $\hat{F}_{0}$ is the same as (3.4). The solution satisfying (2.9) is

$$
F_{0}(\xi)=\frac{2 A \xi}{2 A-\xi}
$$

\section{Theorem 3.2.}

(i) Assume $y(x) \in \mathcal{F}_{A, 1}$ is given by the representation (3.2). If $C_{+} \neq 0$, then the singular points of $y, x_{n}^{+}$, near the boundary $\{x: \arg (2 A x)=\pi / 2\}$ of the sector of analyticity are given asymptotically by

$$
(2 A) x_{n}^{+}=2 n \pi \mathrm{i}-\beta_{1} \ln (2 n \pi \mathrm{i})+\ln \left(C_{+}\right)-\ln (2 A)+o(1), \quad n \rightarrow \infty .
$$

If $C_{-} \neq 0$, then the singular points of $y, x_{n}^{-}$, near the boundary $\{x: \arg (2 A x)=-\pi / 2\}$ of the sector of analyticity are given asymptotically by

$$
(2 A) x_{n}^{-}=-2 n \pi \mathrm{i}-\beta_{1} \ln (-2 n \pi \mathrm{i})+\ln \left(C_{-}\right)-\ln (2 A)+o(1), \quad n \rightarrow \infty .
$$

(ii) Assume $y(x) \in \mathcal{F}_{A, 2}$ is given by the representation (3.3). If $\hat{C}_{+} \neq 0$, then the singular points of $y, \tilde{x}_{n}^{+}$, near the boundary $\{\tilde{x}: \arg (-2 A \tilde{x})=\pi / 2\}$ of the sector of analyticity are given asymptotically by

$$
(-2 A) \tilde{x}_{n}^{+}=2 n \pi \mathrm{i}-\beta_{2} \ln (2 n \pi \mathrm{i})+\ln \left(\hat{C}_{+}\right)-\ln (2 A)+o(1), \quad n \rightarrow \infty .
$$

If $\hat{C}_{-} \neq 0$, then the singular points of $y, \tilde{x}_{n}^{-}$, near the boundary $\{\tilde{x}: \arg (-2 A \tilde{x})=-\pi / 2\}$ of the sector of analyticity are given asymptotically by

$$
(-2 A) \tilde{x}_{n}^{-}=-2 n \pi \mathrm{i}-\beta_{2} \ln (-2 n \pi \mathrm{i})+\ln \left(\hat{C}_{-}\right)-\ln (2 A)+o(1), \quad n \rightarrow \infty .
$$

From Theorem 2.4 we obtain the following results about tritronquée solutions of (1.2):

Theorem 3.3. (1.2) has two tritronquée solutions $y^{+}(x)$ and $y^{-}(x)$ given by

$$
y^{+}(x)=l(x)+h^{+}(2 A x), \quad y^{-}(x)=l(x)+h^{-}(2 A x) .
$$

Let $C_{j}^{t}, 1 \leq j \leq 4$ be as in (2.15). Then

(i) $\mathcal{F}_{A, 1} \cap \mathcal{F}_{A, 2}=\left\{y^{+}, y^{-}\right\}$. 
(ii) For each $\delta>0$ there exists $R>0$ such that $y^{+}(x)$ is analytic in $A^{-1} T_{\delta, R}^{+}$, and $y^{+}$is asymptotic to $y_{0}(x)$ in the sector

$$
\bigcup_{-\frac{\pi}{2}<\phi<\frac{3 \pi}{2}}\left(A^{-1} \mathrm{e}^{\mathrm{i} \phi} \mathbb{R}^{+}\right) .
$$

The singular points of $y^{+}(x), x_{1, n}^{ \pm}$, near the boundary of the sector of analyticity are given asymptotically by

$$
\begin{aligned}
& (2 A) x_{1, n}^{-}=-2 n \pi \mathrm{i}-\beta_{1} \ln (-2 n \pi \mathrm{i})+\ln \left(C_{1}^{t}\right)-\ln (2 A)+o(1), \quad n \rightarrow \infty, \\
& (2 A) x_{1, n}^{+}=-2 n \pi \mathrm{i}+\beta_{2} \ln (2 n \pi \mathrm{i})-\ln \left(C_{4}^{t}\right)+\ln (2 A)+o(1), \quad n \rightarrow \infty .
\end{aligned}
$$

(iii) For each $\delta>0$ there exists $R>0$ such that $y^{-}(x)$ is analytic in $A^{-1} T_{\delta, R}^{-}$, and $y^{-}$is asymptotic to $y_{0}(x)$ in the sector

$$
\bigcup_{-\frac{3 \pi}{2}<\phi<\frac{\pi}{2}}\left(A^{-1} \mathrm{e}^{\mathrm{i} \phi} \mathbb{R}^{+}\right) .
$$

The singular points of $y^{-}(x), x_{2, n}^{ \pm}$, near the boundary of the sector of analyticity are given asymptotically by

$$
\begin{aligned}
& (2 A) x_{2, n}^{-}=2 n \pi \mathrm{i}+\beta_{2} \ln (-2 n \pi \mathrm{i})-\ln \left(C_{3}^{t}\right)+\ln (2 A)+o(1), \quad n \rightarrow \infty, \\
& (2 A) x_{2, n}^{+}=2 n \pi \mathrm{i}-\beta_{1} \ln (2 n \pi \mathrm{i})+\ln \left(C_{2}^{t}\right)-\ln (2 A)+o(1), \quad n \rightarrow \infty .
\end{aligned}
$$

\subsection{Tronquée solutions of $\mathbf{P}_{\text {III }}^{(i i)}$}

If $y(x)$ is a solution of (1.3) which is asymptotic to a formal power series on a ray $d$ which is not an antistokes line, then by dominant balance we have

$$
y(x) \sim l(x), \quad|x| \rightarrow \infty, \quad x \in d,
$$

where

$$
l(x)=A x^{1 / 3}-\frac{\beta}{3 A x^{1 / 3}}
$$

for some $A$ satisfying $A^{3}=1$. Fix an $A$ satisfying $A^{3}=1$ and make the change of variables

$$
w=(27 A / 4)^{1 / 2} x^{2 / 3}, \quad y(x)=x^{1 / 3} h(w)+l(x) .
$$

Then the equation (1.3) is transformed into an equation for $h$ of the form (1.6) with

$$
\beta_{1}=\beta_{2}=\frac{1}{2} .
$$

Let the notations be the same as in Section 2. In view of the transformation (3.7), we denote

$$
\begin{aligned}
& S_{R}^{(0)}:=\left\{x:|x| \geq R, \arg (x) \in\left[-\frac{3 \pi}{4}-\frac{3 \arg A}{4}, \frac{3 \pi}{4}-\frac{3 \arg A}{4}\right]\right\}, \\
& S_{R}^{(1)}:=\left\{x:|x| \geq R, \arg (x) \in\left[\frac{3 \pi}{4}-\frac{3 \arg A}{4}, \frac{9 \pi}{4}-\frac{3 \arg A}{4}\right]\right\}, \\
& S_{R}^{(2)}:=\left\{x:|x| \geq R, \arg (x) \in\left[\frac{9 \pi}{4}-\frac{3 \arg A}{4}, \frac{15 \pi}{4}-\frac{3 \arg A}{4}\right]\right\},
\end{aligned}
$$




$$
S_{R}^{(3)}:=\left\{x:|x| \geq R, \arg (x) \in\left[\frac{15 \pi}{4}-\frac{3 \arg A}{4}, \frac{21 \pi}{4}-\frac{3 \arg A}{4}\right]\right\} .
$$

We notice that for $j \in\{0,2\}, S_{R}^{(j)}$ is mapped under the transformation (3.7) bijectively to the closed sector $\bar{S}_{1} \backslash \mathbb{D}_{R_{0}}$ in the $w$-plane, where $R_{0}=R^{2 / 3}$ (see also Note 2.3); for $j \in\{1,3\}, S_{R}^{(j)}$ is mapped bijectively to the closed sector $\bar{S}_{2} \backslash \mathbb{D}_{R_{0}}$ in the $w$-plane.

\section{Theorem 3.4.}

(i) There is a unique formal power series solution

$$
\tilde{y}_{0}(x)=x^{1 / 3} \sum_{k=0}^{\infty} \frac{y_{0, k}}{x^{2 k / 3}}
$$

to (1.3), where

$$
y_{0,0}=A, \quad y_{0,1}=-\frac{\beta}{3 A} .
$$

(ii) For each $j \in\{0,1,2,3\}$, there is a one-parameter family $\mathcal{F}_{A, j}$ of tronquée solutions of (1.3) in $S_{R}^{(j)}$ where

$$
y(x)=l(x)+x^{1 / 3} h(w), \quad w=K x^{2 / 3}, \quad K=(27 A / 4)^{1 / 2} .
$$

If $j$ is even, then $h(w)$ has the representations

$$
h(w)= \begin{cases}h^{+}(w)+\sum_{k=1}^{\infty} C_{+}^{k} \mathrm{e}^{-k w} \mathcal{L}_{\phi} H_{k}(w), & -\phi \in\left(0, \frac{\pi}{2}\right], \\ h^{-}(w)+\sum_{k=1}^{\infty} C_{-}^{k} \mathrm{e}^{-k w} \mathcal{L}_{\phi} H_{k}(w), & -\phi \in\left[-\frac{\pi}{2}, 0\right) .\end{cases}
$$

If $j$ is odd, then $h(w)$ has the representations

$$
h(w)= \begin{cases}h^{-}(w)+\sum_{k=1}^{\infty} \hat{C}_{+}^{k} \mathrm{e}^{k w} \mathcal{L}_{\phi} \hat{H}_{k}(-w), & -\phi \in\left(0, \frac{\pi}{2}\right], \\ h^{+}(w)+\sum_{k=1}^{\infty} \hat{C}_{-}^{k} \mathrm{e}^{k w} \mathcal{L}_{\phi} \hat{H}_{k}(-w), & -\phi \in\left[-\frac{\pi}{2}, 0\right) .\end{cases}
$$

(iii) Let $y(x)$ be a tronquée solution in $\mathcal{F}_{A, j}$. If $j$ is even, the region of analyticity contains the corresponding branch of $\left(K^{-1} S_{\text {an }}(h)\right)^{2 / 3}$, which contains $S_{R}^{(j)}$ for $R$ large enough. If $j$ is odd, the region of analyticity contains the corresponding branch of $\left(K^{-1} \hat{S}_{\text {an }}(h)\right)^{2 / 3}$, which contains $S_{R}^{(j)}$ for $R$ large enough, and

$$
y(x) \sim \tilde{y}_{0}(x), \quad x \in d, \quad|x| \rightarrow \infty
$$

where $d$ is a ray whose infinite part is contained in the interior of $S_{R}^{(j)}$.

Assume that $y(x)$ is a tronquée solution to (1.3) and $h$ is defined by (3.7). Then $h$ has the representation (3.8) or (3.9). From Theorem 2.4 we obtain information about singularities of $h$. 
Let $\xi_{+}=C_{+} \mathrm{e}^{-w} w^{-1 / 2}, \xi_{-}=C_{-} \mathrm{e}^{-w} w^{-1 / 2}, F_{m}$ and $\hat{F}_{m}$ be as in Section 2. Then the equation satisfied by $F_{0}$ and $\hat{F}_{0}$ is the same

$$
\xi^{2} \frac{\mathrm{d}^{2}}{\mathrm{~d} \xi^{2}} F_{0}(\xi)+\left(\frac{\mathrm{d}}{\mathrm{d} \xi} F_{0}(\xi)\right) \xi-\frac{\left(\frac{\mathrm{d}}{\mathrm{d} \xi} F_{0}(\xi)\right)^{2} \xi^{2}}{A+F_{0}(\xi)}-\frac{\left(A+F_{0}(\xi)\right)^{2}}{3 A}+\frac{1}{3 A\left(A+F_{0}(\xi)\right)}=0
$$

The solution satisfying $(2.9)$ is

$$
F_{0}(\xi)=\frac{36 A^{2} \xi}{(6 A-\xi)^{2}}
$$

\section{Theorem 3.5.}

(i) If $j \in\{0,2\}$, then $h$ has representation (3.8) for a unique pair of constants $\left(C_{+}, C_{-}\right)$. If $C_{+} \neq 0$, then the singular points of $h, w_{n}^{+}$, near the boundary $\{w: \arg w=\pi / 2\}$ of the sector of analyticity are given asymptotically by

$$
w_{n}^{+}=2 n \pi \mathrm{i}-\frac{\ln (2 n \pi \mathrm{i})}{2}+\ln \left(C_{+}\right)-\ln (6 A)+o(1), \quad n \rightarrow \infty .
$$

If $C_{-} \neq 0$, then the singular points of $h, w_{n}^{-}$, near the boundary $\{w: \arg w=-\pi / 2\}$ of the sector of analyticity are given asymptotically by

$$
w_{n}^{-}=-2 n \pi \mathrm{i}-\frac{\ln (-2 n \pi \mathrm{i})}{2}+\ln \left(C_{-}\right)-\ln (6 A)+o(1), \quad n \rightarrow \infty .
$$

(ii) If $j \in\{1,3\}$, then $h$ has representation (3.9) for a unique pair of constants $\left(\hat{C}_{+}, \hat{C}_{-}\right)$. If $\hat{C}_{+} \neq 0$, then the singular points of $h, \tilde{w}_{n}^{+}$, near the boundary $\{\tilde{w}: \arg \tilde{w}=-\pi / 2\}$ of the sector of analyticity are given asymptotically by

$$
\tilde{w}_{n}^{+}=-2 n \pi \mathrm{i}+\frac{\ln (2 n \pi \mathrm{i})}{2}-\ln \left(\hat{C}_{+}\right)+\ln (6 A)+o(1), \quad n \rightarrow \infty .
$$

If $\hat{C}_{-} \neq 0$, then the singular points of $h, \tilde{w}_{n}^{-}$, near the boundary $\{\tilde{w}: \arg \tilde{w}=\pi / 2\}$ of the sector of analyticity are given asymptotically by

$$
\tilde{w}_{n}^{-}=2 n \pi \mathrm{i}+\frac{\ln (-2 n \pi \mathrm{i})}{2}-\ln \left(\hat{C}_{-}\right)+\ln (6 A)+o(1), \quad n \rightarrow \infty .
$$

\section{Theorem 3.6.}

(i) For each $j \in\{0,2\}$ we have a tritronquée solution $y_{j}^{+}$analytic in $S_{R}^{(j)} \cup S_{R}^{(j+1)}$ for $R$ large enough, given by

$$
y_{j}^{+}(x)=l(x)+h^{+}(w) .
$$

(ii) For each $j \in\{1,3\}$ we have a tritronquée solution $y_{j}^{-}$analytic in $S_{R}^{(j)} \cup S_{R}^{(j+1)}$, where $S_{R}^{(4)}=S_{R}^{(0)}$ and $R$ is large enough, given by

$$
y_{j}^{-}(x)=l(x)+h^{-}(w) .
$$

Let $C_{j}^{t}, 1 \leq j \leq 4$ be as in (2.15). Then 
(iii) The singular points of $h^{+}(w), w_{1, n}^{ \pm}$, near the boundary of the sector of analyticity are given asymptotically by

$$
\begin{aligned}
& w_{1, n}^{-}=-2 n \pi \mathrm{i}-\frac{\ln (-2 n \pi \mathrm{i})}{2}+\ln \left(C_{1}^{t}\right)-\ln (6 A)+o(1), \\
& w_{1, n}^{+}=-2 n \pi \mathrm{i}+\frac{\ln (2 n \pi \mathrm{i})}{2}-\ln \left(C_{4}^{t}\right)+\ln (6 A)+o(1) .
\end{aligned}
$$

(iv) The singular points of $h^{-}(w), w_{2, n}^{ \pm}$, near the boundary of the sector of analyticity are given asymptotically by

$$
\begin{aligned}
& w_{2, n}^{-}=2 n \pi \mathrm{i}+\frac{\ln (-2 n \pi \mathrm{i})}{2}-\ln \left(C_{3}^{t}\right)+\ln (6 A)+o(1), \\
& w_{2, n}^{+}=2 n \pi \mathrm{i}-\frac{\ln (2 n \pi \mathrm{i})}{2}+\ln \left(C_{2}^{t}\right)-\ln (6 A)+o(1) .
\end{aligned}
$$

\subsection{Tronquée solutions of $\mathbf{P}_{\mathrm{IV}}$}

By dominant balance we have four possibilities for the leading behavior of $\mathrm{P}_{\mathrm{IV}}$. We shall study them one by one.

$$
y(x) \sim l(x), \quad|x| \rightarrow \infty, \quad x \in d .
$$

\subsubsection{Case 1}

$$
l(x)=-\frac{2 x}{3}+\frac{\alpha}{x} .
$$

Make the change of variables

$$
x=(\sqrt{3} \mathrm{i} w)^{1 / 2}, \quad y(x)=x h(w)+l(x) .
$$

Then the equation (1.1) is transformed into an equation for $h$ of the form (1.6) with

$$
\beta_{1}=\beta_{2}=\frac{1}{2} \text {. }
$$

Let the notations be the same as in Section 2. In view of the transformation (3.12), we denote

$$
\begin{array}{ll}
S_{R}^{(0)}:=\left\{x:|x| \geq R, \arg (x) \in\left[0, \frac{\pi}{2}\right]\right\}, \quad S_{R}^{(1)}:=\left\{x:|x| \geq R, \arg (x) \in\left[\frac{\pi}{2}, \pi\right]\right\},(3.13) \\
S_{R}^{(2)}:=\left\{x:|x| \geq R, \arg (x) \in\left[\pi, \frac{3 \pi}{2}\right]\right\}, & S_{R}^{(3)}:=\left\{x:|x| \geq R, \arg (x) \in\left[\frac{3 \pi}{2}, 2 \pi\right]\right\} .
\end{array}
$$

We notice that for $j \in\{0,2\}, S_{R}^{(j)}$ is mapped under the transformation (3.7) bijectively to the closed sector $\bar{S}_{1} \backslash \mathbb{D}_{R^{2}}$ in the $w$-plane, (see also Note 2.3 ); for $j \in\{1,3\}, S_{R}^{(j)}$ is mapped bijectively to the closed sector $\bar{S}_{2} \backslash \mathbb{D}_{R^{2}}$ in the $w$-plane.

\section{Theorem 3.7.}

(i) There is a formal power series solution of (1.1) of the form

$$
\tilde{y}_{0}(x)=x \sum_{k=0}^{\infty} \frac{y_{0, k}}{x^{2 k}},
$$

where

$$
y_{0,0}=-\frac{2}{3}, \quad y_{0,1}=\alpha .
$$


(ii) For each $j \in\{0,1,2,3\}$, there is a one-parameter family $\mathcal{F}_{A, j}$ of tronquée solutions of (1.1) in $S_{R}^{(j)}$, where

$$
y(x)=l(x)+x h(w), \quad w=\frac{x^{2}}{\sqrt{3} \mathrm{i}} .
$$

If $j$ is even, then $h(w)$ has the representations

$$
h(w)= \begin{cases}h^{+}(w)+\sum_{k=1}^{\infty} C_{+}^{k} \mathrm{e}^{-k w} \mathcal{L}_{\phi} H_{k}(w), & -\phi \in\left(0, \frac{\pi}{2}\right], \\ h^{-}(w)+\sum_{k=1}^{\infty} C_{-}^{k} \mathrm{e}^{-k w} \mathcal{L}_{\phi} H_{k}(w), & -\phi \in\left[-\frac{\pi}{2}, 0\right) .\end{cases}
$$

If $j$ is odd, then $h(w)$ has the representations

$$
h(w)= \begin{cases}h^{-}(w)+\sum_{k=1}^{\infty} \hat{C}_{+}^{k} \mathrm{e}^{k w} \mathcal{L}_{\phi} \hat{H}_{k}(-w), & -\phi \in\left(0, \frac{\pi}{2}\right], \\ h^{+}(w)+\sum_{k=1}^{\infty} \hat{C}_{-}^{k} \mathrm{e}^{k w} \mathcal{L}_{\phi} \hat{H}_{k}(-w), & -\phi \in\left[-\frac{\pi}{2}, 0\right) .\end{cases}
$$

(iii) Let $y(x)$ be a tronquée solution in $\mathcal{F}_{A, j}$. If $j$ is even, the region of analyticity contains the corresponding branch of $\left(\sqrt{3} \mathrm{i} S_{\text {an }}(h)\right)^{1 / 2}$, which contains $S_{R}^{(j)}$ for $R$ large enough. If $j$ is odd, the region of analyticity contains the corresponding branch of $\left(\sqrt{3} \mathrm{i} \hat{S}_{\text {an }}(h)\right)^{1 / 2}$, which contains $S_{R}^{(j)}$ for $R$ large enough, and

$$
y(x) \sim \tilde{y}_{0}(x), \quad x \in d, \quad|x| \rightarrow \infty,
$$

where $d$ is a ray whose infinite part is contained in the interior of $S_{R}^{(j)}$.

Assume that $y(x)$ is a tronquée solution to (1.1) satisfying $y(x) \sim-\frac{2 x}{3}$ and $h$ is defined by (3.12). Then $h$ has representation (3.14) or (3.15). From Theorem 2.4 we obtain information about singularities of $h$. Let $\xi_{+}=C_{+} \mathrm{e}^{-w} w^{-1 / 2}, \xi_{-}=C_{-} \mathrm{e}^{-w} w^{-1 / 2}, F_{m}$ and $\hat{F}_{m}$ be as in Section 2 . Then the equation satisfied by $F_{0}$ and $\hat{F}_{0}$ is the same

$$
\begin{aligned}
\xi^{2} \frac{\mathrm{d}^{2}}{\mathrm{~d} \xi^{2}} F_{0}(\xi)+\xi \frac{\mathrm{d}}{\mathrm{d} \xi} F_{0}(\xi)-\frac{3 \xi^{2}\left(\frac{\mathrm{d}}{\mathrm{d} \xi} F_{0}(\xi)\right)^{2}}{2\left(3 F_{0}(\xi)-2\right)} \\
\quad+\frac{\left(3 F_{0}(\xi)-2\right)^{3}}{24}+\frac{\left(3 F_{0}(\xi)-2\right)^{2}}{3}+\frac{3 F_{0}(\xi)-2}{2}=0 .
\end{aligned}
$$

The solution satisfying $(2.9)$ is

$$
F_{0}(\xi)=\frac{4 \xi}{\xi^{2}+2 \xi+4}
$$

with simple poles at $\xi_{s}^{(1)}=-1-\sqrt{3} \mathrm{i}$ and $\xi_{s}^{(2)}=-1+\sqrt{3} \mathrm{i}$. Hence the statements in Theorems 3.5 and 3.6 hold true for function $h$, with $S_{R}^{(j)}$ as defined in (3.13) and $6 A$ in the formula replaced by $\xi_{s}^{(i)}, i=1$ or $i=2$. 


\subsubsection{Case 2}

$$
l(x)=-2 x-\frac{\alpha}{x} .
$$

Make the change of variables

$$
x=(w)^{1 / 2}, \quad y(x)=x h(w)+l(x) .
$$

Then the equation (1.1) is transformed into an equation for $h$ of the form (1.6) with

$$
\beta_{1}=\alpha+\frac{1}{2}, \quad \beta_{2}=-\alpha+\frac{1}{2} .
$$

Let the notations be the same as in Section 2. In view of the transformation (3.12), we denote

$$
\begin{array}{rlrl}
S^{(0)} & :=\left\{x: \arg (x) \in\left(-\frac{\pi}{4}, \frac{\pi}{4}\right)\right\}, & S^{(1)}:=\left\{x: \arg (x) \in\left(\frac{\pi}{4}, \frac{3 \pi}{4}\right)\right\}, \\
S^{(2)}:=\left\{x: \arg (x) \in\left(\frac{3 \pi}{4}, \frac{5 \pi}{4}\right)\right\}, & S^{(3)}:=\left\{x: \arg (x) \in\left(\frac{5 \pi}{4}, \frac{7 \pi}{4}\right)\right\} .
\end{array}
$$

For $j \in\{0,2\}, S^{(j)}$ is mapped under the transformation (3.7) bijectively to the right half $w$ plane $S_{1}$; for $j \in\{1,3\}, S^{(j)}$ is mapped bijectively to the sector to the left half $w$-plane $S_{2}$.

\section{Theorem 3.8.}

(i) There is a formal power series solution of (1.1) of the form

$$
\tilde{y}_{0}(x)=x \sum_{k=0}^{\infty} \frac{y_{0, k}}{x^{2 k}},
$$

where

$$
y_{0,0}=-2, \quad y_{0,1}=-\alpha .
$$

(ii) For each $j \in\{0,1,2,3\}$, there is a one-parameter family $\mathcal{F}_{A, j}$ of tronquée solutions of (1.1) in $S^{(j)}$, where

$$
y(x)=l(x)+x h(w), \quad w=x^{2} .
$$

If $j$ is even, then $h(w)$ has the representations

$$
h(w)= \begin{cases}h^{+}(w)+\sum_{k=1}^{\infty} C_{+}^{k} \mathrm{e}^{-k w} w^{k M_{1}} \mathcal{L}_{\phi} H_{k}(w), & -\phi \in\left(0, \frac{\pi}{2}\right), \\ h^{-}(w)+\sum_{k=1}^{\infty} C_{-}^{k} \mathrm{e}^{-k w} w^{k M_{1}} \mathcal{L}_{\phi} H_{k}(w), & -\phi \in\left(-\frac{\pi}{2}, 0\right) .\end{cases}
$$

If $j$ is odd, then $h(w)$ has the representations

$$
h(w)= \begin{cases}h^{-}(w)+\sum_{k=1}^{\infty} \hat{C}_{+}^{k} \mathrm{e}^{k w}(-w)^{k M_{2}} \mathcal{L}_{\phi} \hat{H}_{k}(-w), & -\phi \in\left(0, \frac{\pi}{2}\right), \\ h^{+}(w)+\sum_{k=1}^{\infty} \hat{C}_{-}^{k} \mathrm{e}^{k w}(-w)^{k M_{2}} \mathcal{L}_{\phi} \hat{H}_{k}(-w), & -\phi \in\left(-\frac{\pi}{2}, 0\right) .\end{cases}
$$


(iii) Let $y(x)$ be a tronquée solution in $\mathcal{F}_{A, j}$. If $j$ is even, then the region of analyticity contains the corresponding branch of $\left(S_{\mathrm{an}}(h)\right)^{1 / 2}$. If $j$ is odd, then the region of analyticity contains the corresponding branch of $\left(\hat{S}_{\mathrm{an}}(h)\right)^{1 / 2}$, and

$$
y(x) \sim \tilde{y}_{0}(x), \quad x \in d \subset S^{(j)}, \quad|x| \rightarrow \infty .
$$

Assume that $y(x)$ is a tronquée solution to (1.1) satisfying $y(x) \sim-2 x$ and $h$ is defined by (3.18). Then $h$ has representation (3.20) or (3.21). From Theorem 2.4 we obtain information about singularities of $h$. Let $F_{m}$ and $\hat{F}_{m}$ be as in Section 2. Then the equation satisfied by $F_{0}$ and $\hat{F}_{0}$ is the same

$$
\begin{gathered}
\xi^{2} \frac{\mathrm{d}^{2}}{\mathrm{~d} \xi^{2}} F_{0}(\xi)+\xi \frac{\mathrm{d}}{\mathrm{d} \xi} F_{0}(\xi)-\frac{\xi^{2}\left(\frac{\mathrm{d}}{\mathrm{d} \xi} F_{0}(\xi)\right)^{2}}{2\left(F_{0}(\xi)-2\right)}-\frac{3\left(F_{0}(\xi)-2\right)^{3}}{8} \\
-\left(F_{0}(\xi)-2\right)^{2}-\frac{F_{0}(\xi)-2}{2}=0 .
\end{gathered}
$$

The solution satisfying $(2.9)$ is

$$
F_{0}(\xi)=\frac{2 \xi}{\xi+2}
$$

with a simple pole at $\xi_{s}=-2$.

\section{Theorem 3.9.}

( $i)$ If $j \in\{0,2\}$, then $h$ has representation (3.20) for a unique pair of constants $\left(C_{+}, C_{-}\right)$. If $C_{+} \neq 0$, then the singular points of $h$, at $w_{n}^{+}$, near the boundary $\{w: \arg w=\pi / 2\}$ of the sector of analyticity are given asymptotically by

$$
w_{n}^{+}=2 n \pi \mathrm{i}-(\alpha+1 / 2) \ln (2 n \pi \mathrm{i})+\ln \left(C_{+}\right)-\ln (-2)+o(1), \quad n \rightarrow \infty .
$$

If $C_{-} \neq 0$, then the singular points of $h, w_{n}^{-}$, near the boundary $\{w: \arg w=-\pi / 2\}$ of the sector of analyticity are given asymptotically by

$$
w_{n}^{-}=-2 n \pi \mathrm{i}-(\alpha+1 / 2) \ln (-2 n \pi \mathrm{i})+\ln \left(C_{-}\right)-\ln (-2)+o(1), \quad n \rightarrow \infty .
$$

(ii) If $j \in\{1,3\}$, then $h$ has representation (3.21) for a unique pair of constants $\left(\hat{C}_{+}, \hat{C}_{-}\right)$. If $\hat{C}_{+} \neq 0$, then the singular points of $h, \tilde{w}_{n}^{+}$, near the boundary $\{\tilde{w}: \arg \tilde{w}=-\pi / 2\}$ of the sector of analyticity are given asymptotically by

$$
\tilde{w}_{n}^{+}=-2 n \pi \mathrm{i}+(-\alpha+1 / 2) \ln (2 n \pi \mathrm{i})-\ln \left(\hat{C}_{+}\right)+\ln (-2)+o(1), \quad n \rightarrow \infty .
$$

If $\hat{C}_{-} \neq 0$, then the singular points of $h, \tilde{w}_{n}^{-}$, near the boundary $\{\tilde{w}: \arg \tilde{w}=\pi / 2\}$ of the sector of analyticity are given asymptotically by

$$
\tilde{w}_{n}^{-}=2 n \pi \mathrm{i}+(-\alpha+1 / 2) \ln (-2 n \pi \mathrm{i})-\ln \left(\hat{C}_{-}\right)+\ln (-2)+o(1), \quad n \rightarrow \infty .
$$

Denote

$$
\begin{aligned}
T_{\delta, R}^{(0)} & :=\left\{w:|w|>R, \arg (w) \in\left[-\frac{\pi}{4}+\delta, \frac{3 \pi}{4}-\delta\right]\right\}, \\
T_{\delta, R}^{(1)} & :=\left\{w:|w|>R, \arg (w) \in\left[\frac{\pi}{4}+\delta, \frac{5 \pi}{4}-\delta\right]\right\}, \\
T_{\delta, R}^{(2)} & :=\left\{w:|w|>R, \arg (w) \in\left[\frac{3 \pi}{4}+\delta, \frac{7 \pi}{4}-\delta\right]\right\}, \\
T_{\delta, R}^{(3)} & :=\left\{w:|w|>R, \arg (w) \in\left[-\frac{3 \pi}{4}+\delta, \frac{\pi}{4}-\delta\right]\right\} .
\end{aligned}
$$




\section{Theorem 3.10.}

(i) Let $j \in\{0,2\}$. For each $\delta>0$ there exists $R$ large enough such that we have a tritronquée solution $y_{j}^{+}$analytic in $T_{\delta, R}^{(j)}$ given by

$$
y_{j}^{+}(x)=-2 x+\frac{\alpha}{x}+h^{+}\left(x^{2}\right) .
$$

(ii) Let $j \in\{1,3\}$. For each $\delta>0$ there exists $R$ large enough such that we have a tritronquée solution $y_{j}^{-}$analytic in $T_{\delta, R}^{(j)}$ given by

$$
y_{j}^{-}(x)=-2 x+\frac{\alpha}{x}+h^{-}\left(x^{2}\right) .
$$

Let $C_{j}^{t}, 1 \leq j \leq 4$ be as in (2.15). Then

(iii) The singular points of $h^{+}(w), w_{1, n}^{ \pm}$, near the boundary of the sector of analyticity are given asymptotically by

$$
\begin{aligned}
& w_{1, n}^{-}=-2 n \pi \mathrm{i}-(\alpha+1 / 2) \ln (-2 n \pi \mathrm{i})+\ln \left(C_{1}^{t}\right)-\ln (-2)+o(1), \\
& w_{1, n}^{+}=-2 n \pi \mathrm{i}+(-\alpha+1 / 2) \ln (2 n \pi \mathrm{i})-\ln \left(C_{4}^{t}\right)+\ln (-2)+o(1) .
\end{aligned}
$$

(iv) The singular points of $h^{-}(w), w_{2, n}^{ \pm}$, near the boundary of the sector of analyticity are given asymptotically by

$$
\begin{aligned}
& w_{2, n}^{-}=2 n \pi \mathrm{i}+(-\alpha+1 / 2) \ln (-2 n \pi \mathrm{i})-\ln \left(C_{3}^{t}\right)+\ln (-2)+o(1), \\
& w_{2, n}^{+}=2 n \pi \mathrm{i}-(\alpha+1 / 2) \ln (2 n \pi \mathrm{i})+\ln \left(C_{2}^{t}\right)-\ln (-2)+o(1) .
\end{aligned}
$$

\subsubsection{Case 3}

$$
l(x)=\frac{A}{x}+\frac{\alpha A+\beta}{2 x^{3}}, \quad A^{2}=-b / 2 .
$$

Make the change of variables

$$
x=(w)^{1 / 2}, \quad y(x)=x^{-1} h(w)+l(x) .
$$

Then the equation (1.1) is transformed into an equation for $h$ of the form (1.6) with

$$
\beta_{1}=-\frac{\alpha}{2}+\frac{3 A}{2}, \quad \beta_{2}=\frac{\alpha}{2}-\frac{3 A}{2} .
$$

Let the notations be as in Section $2, S^{(j)}$ be as in (3.19) and $T_{\delta, R}^{(j)}$ be as in (3.24).

\section{Theorem 3.11.}

(i) There is a formal power series solution of (1.1) of the form

$$
\tilde{y}_{0}(x)=\frac{1}{x} \sum_{k=0}^{\infty} \frac{y_{0, k}}{x^{2 k}},
$$

where

$$
y_{0,0}=A, \quad y_{0,1}=\frac{\alpha A+\beta}{2} .
$$


(ii) For each $j \in\{0,1,2,3\}$, there is a one-parameter family $\mathcal{F}_{A, j}$ of tronquée solutions of (1.1) in $S^{(j)}$, where

$$
y(x)=l(x)+x^{-1} h(w), \quad w=x^{2} .
$$

If $j$ is even, then $h(w)$ has the representations

$$
h(w)= \begin{cases}h^{+}(w)+\sum_{k=1}^{\infty} C_{+}^{k} \mathrm{e}^{-k w} w^{k M_{1}} \mathcal{L}_{\phi} H_{k}(w), & -\phi \in\left(0, \frac{\pi}{2}\right), \\ h^{-}(w)+\sum_{k=1}^{\infty} C_{-}^{k} \mathrm{e}^{-k w} w^{k M_{1}} \mathcal{L}_{\phi} H_{k}(w), & -\phi \in\left(-\frac{\pi}{2}, 0\right) .\end{cases}
$$

If $j$ is odd, then $h(w)$ has the representations

$$
h(w)= \begin{cases}h^{-}(w)+\sum_{k=1}^{\infty} \hat{C}_{+}^{k} \mathrm{e}^{k w}(-w)^{k M_{2}} \mathcal{L}_{\phi} \hat{H}_{k}(-w), & -\phi \in\left(0, \frac{\pi}{2}\right), \\ h^{+}(w)+\sum_{k=1}^{\infty} \hat{C}_{-}^{k} \mathrm{e}^{k w}(-w)^{k M_{2}} \mathcal{L}_{\phi} \hat{H}_{k}(-w), & -\phi \in\left(-\frac{\pi}{2}, 0\right) .\end{cases}
$$

(iii) Let $y(x)$ be a tronquée solution in $\mathcal{F}_{A, j}$. If $j$ is even, then the region of analyticity contains the corresponding branch of $\left(S_{\mathrm{an}}(h)\right)^{1 / 2}$. If $j$ is odd, then the region of analyticity contains the corresponding branch of $\left(\hat{S}_{\mathrm{an}}(h)\right)^{1 / 2}$, and

$$
y(x) \sim \tilde{y}_{0}(x), \quad x \in d \subset S^{(j)}, \quad|x| \rightarrow \infty .
$$

\section{Theorem 3.12.}

(i) Let $j \in\{0,2\}$. For each $\delta>0$ there exists $R$ large enough such that we have a tritronquée solution $y_{j}^{+}$analytic in $T_{\delta, R}^{(j)}$ given by

$$
y_{j}^{+}(x)=\frac{A}{x}+\frac{\alpha A+\beta}{2 x^{3}}+h^{+}\left(x^{2}\right) .
$$

(ii) Let $j \in\{1,3\}$. For each $\delta>0$ there exists $R$ large enough such that we have a tritronquée solution $y_{j}^{-}$analytic in $T_{\delta, R}^{(j)}$ given by

$$
y_{j}^{-}(x)=\frac{A}{x}+\frac{\alpha A+\beta}{2 x^{3}}+h^{-}\left(x^{2}\right) .
$$

Note 3.13. In this case, the corresponding $F_{0}$ and $\hat{F}_{0}$ turn out to be $\xi$, which yield no singularities for $h$. However, it does not imply that the poles are nonexistent. More research needs to be done for this case.

\section{Proofs and further results}

\subsection{Proof of Proposition 2.1}

Let $h$ and $\mathbf{u}$ be as defined in Section 1. We have a system of differential equations (1.8) for $\mathbf{u}$. It is known (see $[3,4,6]$ ) that it admits transseries solutions (i.e., formal exponential power series solutions) of the form

$$
\tilde{\mathbf{u}}(w)=\tilde{\mathbf{u}}_{0}(w)+\sum_{k=1}^{\infty} C^{k} \mathrm{e}^{-k w} w^{-\beta_{1} k} \tilde{\mathbf{u}}_{k}(w),
$$


where $\tilde{\mathbf{u}}_{0}(w)$ and $\tilde{\mathbf{u}}_{k}(w)$ are formal power series in $w^{-1}$, namely

$$
\tilde{\mathbf{u}}_{k}(w)=\sum_{r=0}^{\infty} \frac{\mathbf{u}_{k, r}}{w^{r}}, \quad k \geq 1, \quad \tilde{\mathbf{u}}_{0}(w)=\sum_{r=2}^{\infty} \frac{\mathbf{u}_{0, r}}{w^{r}} .
$$

Also, $\tilde{\mathbf{u}}_{0}(w)$ is the unique power series solution of (1.8). The coefficients in the series $\tilde{\mathbf{u}}_{k}$ can be determined by substitution of the formal exponential power series $\tilde{\mathbf{u}}(w)$ into (4.1) and identification of each coefficient of $\mathrm{e}^{-k w}$. Proposition 2.1 is then obtained through (1.7). Furthermore,

$$
\tilde{h}_{0}(w)=\mathbf{r}_{1} \cdot \tilde{\mathbf{u}}_{0}(w), \quad \tilde{s}_{k}(w)=\mathbf{r}_{1} \cdot \tilde{\mathbf{u}}_{k}(w), \quad \mathbf{r}_{1}=\left[\begin{array}{ll}
1-\frac{\beta_{1}}{2 w} & 1+\frac{\beta_{2}}{2 w}
\end{array}\right]
$$

\subsection{Proof of Theorem 2.2}

Let $d=\mathrm{e}^{\mathrm{i} \theta} \mathbb{R}^{+}$with $\cos \theta>0$, and let $\mathbf{u}$ be a solution to (1.8) on $d$ for $w$ large enough, satisfying

$$
\mathbf{u}(w) \rightarrow \mathbf{0}, \quad w \in d, \quad|w| \rightarrow \infty .
$$

Theorem 3 in [4], Theorem 16, Lemma 17 and Theorem 19 in [6] imply the following results:

\section{Proposition 4.1.}

(i) For any $d^{\prime}=\mathrm{e}^{\mathrm{i} \theta^{\prime}} \mathbb{R}^{+}$where $\cos \theta^{\prime}>0$, the solution $\mathbf{u}(w)$ is analytic on $d^{\prime}$ for $w$ large enough and $\mathbf{u} \sim \tilde{\mathbf{u}}_{0}(w)$ on $d^{\prime}$.

(ii) Given $\phi \in\left(-\frac{\pi}{2}, 0\right) \cup\left(0, \frac{\pi}{2}\right)$, there exists a unique constant $C(\phi)$ such that $\mathbf{u}$ has the following representation:

$$
\mathbf{u}(w)=\mathcal{L}_{\phi} \mathbf{U}_{0}(w)+\sum_{k=1}^{\infty}(C(\phi))^{k} \mathrm{e}^{-k w} w^{k M_{1}} \mathcal{L}_{\phi} \mathbf{U}_{k}(w)
$$

where

$$
\mathbf{U}_{0}=\mathcal{B} \tilde{\mathbf{u}}_{0}, \quad \mathbf{U}_{k}=\mathcal{B}\left(w^{-k \beta_{1}-k M_{1}} \tilde{\mathbf{u}}_{k}\right), \quad k=1,2, \ldots,
$$

where for each $k \geq 1, \mathbf{U}_{k}$ is analytic in the Riemann surface of $\mathbb{C} \backslash\left(\mathbb{Z}^{+} \cup \mathbb{Z}^{-}\right)$, and the branch cut for $\mathbf{U}_{k}$ is chosen to be $(-\infty, 0]$. The function $C(\phi)$ is constant on $\left(-\frac{\pi}{2}, 0\right)$ and also constant on $\left(0, \frac{\pi}{2}\right)$.

(iii) Let $\epsilon$ be small. There exist $\delta, R>0$ such that $\mathbf{u}(w)$ is analytic on

$$
S_{a n, \epsilon}(\mathbf{u}(w))=S_{\epsilon}^{+} \cup S_{\epsilon}^{-},
$$

where $S^{ \pm}$is as defined in $(2.5)$.

We now return to the proof of Theorem 2.2. Assume that $h(w)$ is a solution of (1.6) on $d=\mathrm{e}^{\mathrm{i} \phi} \mathbb{R}^{+}$with $\cos \phi>0$ for $|w|>w_{0}$, where $w_{0}>0$ is large enough. Without loss of generality we may assume that $w_{0}>\frac{\sqrt{\left|\beta_{1} \beta_{2}\right|}}{2}$. Thus the vector function $\mathbf{u}(w)$ defined by

$$
\mathbf{u}(w)=\left[\begin{array}{cc}
1-\frac{\beta_{1}}{2 w} & 1+\frac{\beta_{2}}{2 w} \\
-1-\frac{\beta_{1}}{2 w} & 1-\frac{\beta_{2}}{2 w}
\end{array}\right]^{-1}\left[\begin{array}{c}
h(w) \\
h^{\prime}(w)
\end{array}\right]
$$

is a solution of the differential system (1.8), and $h(w)=\mathbf{r}_{1} \cdot \mathbf{u}(w)$. 
Next we use the basic properties (see Lemmas A.1 and A.2) of the operators $\mathcal{B}$ and $\mathcal{L}_{\phi}$ and obtain the following

$$
\begin{aligned}
& \mathcal{L}_{\phi} \mathcal{B}\left(\mathbf{r}_{1} \cdot \tilde{\mathbf{u}}_{0}\right)=\mathbf{r}_{1} \cdot \mathcal{L}_{\phi} \mathcal{B}\left(\tilde{\mathbf{u}}_{0}\right)=\mathbf{r}_{1} \cdot \mathcal{L}_{\phi} \mathbf{U}_{0} \\
& \mathcal{L}_{\phi} \mathcal{B}\left(w^{-k \beta_{1}-k M_{1}} \mathbf{r}_{1} \cdot \tilde{\mathbf{u}}_{k}\right)=\mathbf{r}_{1} \cdot \mathcal{L}_{\phi} \mathcal{B}\left(w^{-k \beta_{1}-k M_{1}} \tilde{\mathbf{u}}_{k}\right)=\mathbf{r}_{1} \cdot \mathcal{L}_{\phi} \mathbf{U}_{k} .
\end{aligned}
$$

By Proposition 4.1(i), given a ray $d^{\prime}$ in the right half $w$-plane, $h(w)=\mathbf{r}_{1} \cdot \mathbf{u}(w)$ is analytic on $d^{\prime}$ for $|w|$ large enough and is asymptotic to $\tilde{h}_{0}(w)=\mathbf{r}_{1} \cdot \tilde{\mathbf{u}}_{0}(w)$ on $d^{\prime}$. From the representations (4.3), (4.4) in Proposition 4.1(ii) of $\mathbf{u}(w)$ and (4.6) we obtain the representations for $h(w)=\mathbf{r}_{1} \cdot \mathbf{u}(w)$ as in (2.2) and (2.3). For $|w|$ large enough, $h(w)$ is analytic where $\mathbf{u}(w)$ is analytic, hence Proposition 4.1(iii) implies Theorem 2.2(ii). Thus Theorem 2.2 is proved.

\subsection{Proof of Theorem 2.4}

Let $h(w)$ and $\mathbf{u}(w)$ be as in the proof of Theorem 2.2. $h(w)$ has representations (2.2) and (2.3). We will consider the case $C_{+} \neq 0$ and prove (i) and (ii). The statements (iii) and (iv) about the case $C_{-} \neq 0$ follow by symmetry.

By Theorem 1 in [6], there exists $\delta_{1}>0$ such that for $\left|\xi_{+}\right|<\delta_{1}$ the power series

$$
\mathbf{G}_{m}\left(\xi_{+}\right)=\sum_{k=0}^{\infty} \xi_{+}^{k} \mathbf{u}_{k, m}, \quad m=0,1,2, \ldots
$$

converges, where $\mathbf{G}_{0}$ satisfies

$$
\mathbf{G}_{0}=\mathbf{0}, \quad \mathbf{G}_{0}^{\prime}=\mathbf{e}_{1} .
$$

Furthermore,

$$
\mathbf{u}(w) \sim \sum_{m=0}^{\infty} w^{-m} \mathbf{G}_{m}\left(\xi_{+}(w)\right), \quad|w| \rightarrow \infty
$$

holds uniformly in

$$
\mathcal{S}_{\delta_{1}}=\left\{w: \arg (w) \in\left(-\frac{\pi}{2}+\delta, \frac{\pi}{2}+\delta\right),\left|\xi_{+}(w)\right|<\delta_{1}\right\}
$$

By Theorem 2 in [6], for $R$ large enough and $\delta, \epsilon$ small enough, $\mathbf{u}(w)$ is analytic in $\mathcal{D}_{w}^{+}($see $(2.8))$. Also, the asymptotic representation (4.7) holds in $\mathcal{D}_{w}^{+}$. Moreover, if $\mathbf{G}_{0}$ has an isolated singularity at $\xi_{s}$, then $\mathbf{u}(w)$ is singular at a distance at most $o(1)$ of $w_{n}^{+}$given in $(2.10)$, as $w_{n}^{+} \rightarrow \infty$. Since $h(w)=\mathbf{r}_{1} \cdot \mathbf{u}(w)$, Theorem 2.4(i) follows from the results cited.

Assume $|w|>\sqrt{\left|\beta_{1} \beta_{2}\right|} / 2$. Both (1.7) and (4.5) hold. While (1.7) implies that $h$ is analytic at least where $\mathbf{u}$ is analytic, (4.5) implies that $h$ is singular where $\mathbf{u}$ is singular. Thus the asymptotic position of singularities, i.e., poles of $h(w)$ is the same as that of $\mathbf{u}(w)$, which is presented in equation (2.10). Thus Theorem 2.4(ii) is proved.

\subsection{Proof of Corollary 2.5}

Let the notations be the same as in Section 2.2. First we point out some properties of $\mathbf{U}_{0}(p)$. See also [3].

We apply formal inverse Laplace transform to the system (1.8). To be precise, assume the analytic function $\mathbf{g}(w, \mathbf{u})$ has the Taylor expansion at $(\infty, \mathbf{0})$ as follows

$$
\mathbf{g}(w, \mathbf{u})=\sum_{m \geq 0 ;|1| \geq 0} \mathbf{g}_{m, \mathbf{l}} w^{-m} \mathbf{u}^{\mathbf{l}}, \quad\left|w^{-1}\right|<\xi_{0}, \quad|\mathbf{u}|<\xi_{0} .
$$


Note that by assumption $\mathbf{g}_{m, \mathbf{l}}=\mathbf{0}$ if $|\mathbf{l}| \leq 1$ and $m \leq 1$. Denote $\mathbf{U}=\mathcal{L}^{-1} \mathbf{u}$. Then the formal inverse Laplace transform of the differential system (1.8) is the system of convolution equations

$$
-p \mathbf{U}(p)=-\left[\hat{\Lambda} \mathbf{U}(p)+\hat{B} \int_{0}^{p} \mathbf{U}(s) \mathrm{d} s\right]+\mathcal{N}(\mathbf{U})(p),
$$

where

$$
\mathcal{N}(\mathbf{U})(p)=\sum_{m=2}^{\infty} \frac{\mathbf{g}_{m, \mathbf{0}}}{(m-1) !} p^{m-1}+\sum_{|\mathbf{1}| \geq 2} \mathbf{g}_{0, \mathbf{l}} \mathbf{U}^{* \mathbf{1}}+\sum_{|\mathbf{l}| \geq 1}\left(\sum_{m=1}^{\infty} \frac{\mathbf{g}_{m, \mathbf{l}}}{(m-1) !} p^{m-1}\right) * \mathbf{U}^{* \mathbf{l}} .
$$

Let $\mathbf{v}(p)=\left(v_{1}(p), \ldots, v_{n}(p)\right)$ be an $n$-dimensional complex vector function, $f(p)$ be a locally integrable complex function and $\mathbf{l}=\left(l_{1}, \ldots, l_{n}\right)$ be an $n$-dimensional multi-index. Then

$$
\mathbf{v}^{* 1}:=v_{1}^{* l_{1}} * v_{2}^{* l_{2}} * \cdots * v_{n}^{* l_{n}}, \quad(\mathbf{v} * f)(p) \in \mathbb{C}^{n}, \quad(\mathbf{v} * f)_{j}=v_{j} * f, \quad j=1, \ldots, n .
$$

We gather the following facts about $\mathbf{U}_{0}$.

\section{Proposition 4.2.}

(i) Let $K \in \mathcal{O}$ be a closed set such that for every point $p \in K$, the line segment connecting the origin and $p$ is contained in $K$. Then $\mathbf{U}_{0}$ is the unique solution to (4.8) in $K$.

(ii) $\mathbf{U}_{0}=\mathcal{B} \tilde{\mathbf{u}}_{0} . \quad \mathbf{U}_{0}$ is analytic in the domain $\mathcal{O}=\mathbb{C} \backslash[(\infty,-1] \cup[1, \infty)]$, and is Laplace transformable along any ray $\mathrm{e}^{\mathrm{i} \phi} \mathbb{R}^{+}$contained in $\mathcal{O} . \mathcal{L}_{\phi} \mathbf{U}_{0}$ is a solution of (4.8) for each $\phi$ such that $|\cos (\phi)|<1$.

(iii) Let $K$ be as in $(i)$. There exists $b_{K}>0$ large enough such that

$$
\sup _{p \in K} \int_{[0, p]}\left|\mathbf{U}_{0}(s)\right| \mathrm{e}^{-b_{K}|s|}|\mathrm{d} s|<\infty .
$$

Proposition 4.2(i) and (ii) come from Proposition 6 in [3]. Although (iii) is not stated explicitly in [3], it can be easily obtained by the same approach used to prove Proposition 6 . Let $K$ be as in (i). Consider the Banach space

$$
L_{\text {ray }}(K):=\{\mathbf{f}: \mathbf{f} \text { is locally integrable on }[0, p] \text { for each } p \in K\}
$$

equipped with the norm $\|\cdot\|_{b, K}$ defined by

$$
\|\mathbf{f}\|_{b, K}:=\sup _{p \in K} \int_{[0, p]}\|\mathbf{f}(s)\| \mathrm{e}^{-b|s|}|\mathrm{d} s|,
$$

where $\|\mathbf{f}(s)\|=\max \left\{\left|f_{1}(s)\right|,\left|f_{2}(s)\right|\right\}$. We can show that for $b$ large enough, the operator

$$
\mathcal{N}_{1}:=\mathbf{U}(p) \mapsto(\hat{\Lambda}-p I)^{-1}\left(-\hat{B} \int_{0}^{p} \mathbf{U}(s) \mathrm{d} s+\mathcal{N}(\mathbf{U})(p)\right)
$$

is contractive in the closed ball $\mathcal{S}:=\left\{\mathbf{f} \in L_{\text {ray }}(K):\|\mathbf{f}\|_{b, K} \leq \delta\right\}$ of $L_{\text {ray }}(K)$ if $\delta$ is small enough. By contractive mapping theorem there is a unique solution of $\mathcal{N}_{1} \mathbf{U}=\mathbf{U}$ in $\mathcal{S}$, namely $\mathbf{U}_{0}$ by uniqueness of the solution. Using integration by parts and (iii) we have the following:

\section{Corollary 4.3.}

(i) If $\phi \in(0, \pi)$ or $\phi \in(-\pi, 0), \mathcal{L}_{\phi} \mathbf{U}_{0}(w)$ is analytic (at least) in the region

$$
\mathcal{A}_{\phi}:=\{w:|w| \cos (\phi+\arg (w))>b\},
$$

where $b=b_{K}$ is as in Proposition $4.2($ iii $)$ with $K=\mathrm{e}^{\mathrm{i} \phi} \mathbb{R}^{+}$. 
(ii) If $0<\phi_{1}<\phi_{2}<\pi$ or $0<-\phi_{1}<-\phi_{2}<\pi$, then $\mathcal{L}_{\phi_{1}} \mathbf{U}_{0}$ and $\mathcal{L}_{\phi_{2}} \mathbf{U}_{0}$ are analytic continuations of each other.

Since $H_{0}=\mathcal{B} \tilde{h}_{0}$, by $(4.2)$ and Lemma A.1 we have

$$
\begin{aligned}
H_{0}(p) & =\left(\mathcal{B} u_{0,1}\right)(p)+\left(\mathcal{B} u_{0,2}\right)(p)-\frac{\beta_{1}}{2}\left[1 *\left(\mathcal{B} u_{0,1}\right)\right](p)+\frac{\beta_{2}}{2}\left[1 *\left(\mathcal{B} u_{0,2}\right)\right](p) \\
& =U_{0,1}(p)+U_{0,2}(p)-\frac{\beta_{1}}{2}\left(1 * U_{0,1}\right)(p)+\frac{\beta_{2}}{2}\left(1 * U_{0,2}\right)(p),
\end{aligned}
$$

where $u_{0, i}, i=1,2$, is the $i$-th component of the vector function $\mathbf{u}_{0}$ and $U_{0, i}, i=1,2$, is the $i$-th component of $\mathbf{U}_{0}$. It is clear from (4.9) that Proposition 4.2(iii) and Corollary 4.3 hold with $\mathbf{U}_{0}$ replaced by $H_{0}$.

Merely by $\hat{H}_{0}(p)=-H_{0}(-p)$ and Corollary $4.3(\mathrm{ii})$ with $\mathbf{U}_{0}$ replaced by $H_{0}$ we obtain Corollary 2.5(i). Moreover, both $h^{+}$and $h^{-}$are special cases of tronquée solutions, thus Theorems 2.2 and 2.4 apply. $h^{+}$is analytic at least on $S_{\text {an }}\left(h^{+}\right) \cup\left(-S_{\text {an }}\left(\hat{h}^{+}\right)\right)$and $h^{-}$is analytic at least on $S_{\text {an }}\left(h^{-}\right) \cup\left(-S_{\text {an }}\left(\hat{h}^{-}\right)\right)$. We also obtain the asymptotic position of singularities of the tritronquée solutions as in Corollary 2.5(ii).

\subsection{Proof of the results in Section 3}

Once we have the normalizations in the form of (1.6) of the equations (1.2), (1.3) and (1.1), the results in Section 3 follow from the results in Section 2. Here we present the details of finding solutions to (3.4), (3.10), (3.16) and (3.22) satisfying (2.9).

\subsubsection{Solving (3.4)}

Make the substitution $Q(s)=A+F_{0}\left(\mathrm{e}^{s}\right)$ then (3.4) transforms into

$$
\frac{\mathrm{d}^{2}}{\mathrm{~d} s^{2}} Q(s)-\frac{\left(\frac{\mathrm{d}}{\mathrm{d} s} Q(s)\right)^{2}}{Q(s)}-\frac{(Q(s))^{3}}{4 A^{2}}+\frac{1}{4 A^{2} Q(s)}=0 .
$$

Multiplying both sides by $1 / Q(s)$ we obtain

$$
\frac{\mathrm{d}}{\mathrm{d} s}\left(\frac{Q^{\prime}(s)}{Q(s)}\right)=\frac{1}{4 A^{2}}\left(Q^{2}(s)-\frac{1}{Q^{2}(s)}\right) .
$$

Multiplying both sides by $2 Q^{\prime}(s) / Q(s)$ and integrating with respect to $s$ we have

$$
\begin{aligned}
& \left(\frac{Q^{\prime}(s)}{Q(s)}\right)^{2}=\frac{1}{4 A^{2}}\left(Q^{2}(s)+\frac{1}{Q^{2}(s)}+C_{1}\right), \quad \text { i.e., } \\
& \left(Q^{\prime}(s)\right)^{2}=\frac{1}{4 A^{2}}\left(Q^{4}(s)+C_{1} Q^{2}(s)+1\right) .
\end{aligned}
$$

By a linear transformation $Q(s)=\tilde{Q}(s) /(2 A),(4.10)$ is reduced to the Jacobi normal form which is solved by Jacobi elliptic functions unless $C_{1} \in\{-2,2\}$. Since $Q(s)=A+F_{0}\left(\mathrm{e}^{s}\right)$ and $F_{0}(0)=0$ (see (2.9)), the solution we look for cannot be an elliptic function. Moreover, as $\operatorname{Re}(s) \rightarrow-\infty$, $Q(s) \rightarrow A$ implies $Q^{\prime}(s) \rightarrow 0$, so (4.10) needs to be of the form

$$
\left(Q^{\prime}(s)\right)^{2}=\frac{1}{4 A^{2}}\left(Q^{2}(s)-A^{2}\right)^{2} .
$$

The solution satisfying $Q(s) \rightarrow A$ as $\operatorname{Re}(s) \rightarrow-\infty$ is

$$
Q(s)=A \cdot \frac{C_{2}-\mathrm{e}^{s}}{C_{2}+\mathrm{e}^{s}}, \quad C_{2} \neq 0 .
$$


Thus the solution to (3.4) is

$$
F_{0}(\xi)=-\frac{2 A \xi}{C_{2}+\xi}
$$

Hence the solution to (3.4) satisfying (2.9) is

$$
F_{0}(\xi)=\frac{2 A \xi}{2 A-\xi}
$$

\subsubsection{Solving (3.10)}

Make the substitution $Q(s)=A+F_{0}\left(\mathrm{e}^{s}\right)$ then (3.10) transforms into

$$
\frac{\mathrm{d}^{2}}{\mathrm{~d} s^{2}} Q(s)-\frac{\left(\frac{\mathrm{d}}{\mathrm{d} s} Q(s)\right)^{2}}{Q(s)}-\frac{(Q(s))^{2}}{3 A}+\frac{1}{3 A Q(s)}=0 .
$$

Multiplying both sides by $1 / Q(s)$ we obtain

$$
\frac{\mathrm{d}}{\mathrm{d} s}\left(\frac{Q^{\prime}(s)}{Q(s)}\right)=\frac{1}{3 A}\left(Q(s)-\frac{1}{Q^{2}(s)}\right) .
$$

Multiplying both sides by $2 Q^{\prime}(s) / Q(s)$ and integrating with respect to $s$ we have

$$
\begin{aligned}
& \left(\frac{Q^{\prime}(s)}{Q(s)}\right)^{2}=\frac{1}{3 A}\left(2 Q(s)+\frac{1}{Q^{2}(s)}+C_{1}\right), \quad \text { i.e., } \\
& \left(Q^{\prime}(s)\right)^{2}=\frac{1}{3 A}\left(2 Q^{3}(s)+C_{1} Q^{2}(s)+1\right) .
\end{aligned}
$$

Notice that if the equation $2 x^{3}+C_{1} x^{2}+1=0$ has three distinct roots then (4.11) is known to have Weierstrass $\wp$-functions as general solutions, in which case the corresponding $F_{0}(\xi)=Q(\ln \xi)-A$ fails to satisfy the condition (2.9). Hence $C_{1}$ must be such that the equation $2 x^{3}+C_{1} x^{2}+1=0$ has a multiple root. Denote the multiple root by $r_{1}$. Then

$$
2 x^{3}+C_{1} x^{2}+1=2\left(x-r_{1}\right)^{2}\left(x-r_{2}\right) .
$$

Then we obtain

$$
r_{1}=-2 r_{2}, \quad r_{1}^{3}=1, \quad C_{1}=-3 r_{1} .
$$

Since $Q(s) \rightarrow A$ as $\operatorname{Re}(s) \rightarrow-\infty, Q^{\prime}(s) \rightarrow 0$. Hence $r_{1}=A$ or $r_{2}=A$. We knew from the normalization (see (3.6)) that $A^{3}=1$. Thus $r_{1}=A, r_{2}=-A / 2$, and $C_{1}=-3 A$. Hence (4.11) is of the form

$$
\left(Q^{\prime}(s)\right)^{2}=\frac{2}{3 A}(Q(s)-A)^{2}\left(Q(s)+\frac{A}{2}\right) .
$$

The solution to $(4.12)$ is

$$
Q(s)=-\frac{A}{2}+\frac{3 A}{2}\left(\frac{C_{2}-\mathrm{e}^{s}}{C_{2}+\mathrm{e}^{s}}\right)^{2} .
$$

Hence the solution of (3.10) satisfying (2.9) is

$$
F_{0}(\xi)=\frac{36 A^{2} \xi}{(6 A-\xi)^{2}}
$$




\subsubsection{Solving (3.16)}

Make the substitution $Q(s)=F_{0}\left(\mathrm{e}^{s}\right)-2 / 3$ then (3.16) transforms into

$$
\frac{\mathrm{d}^{2}}{\mathrm{~d} s^{2}} Q(s)-\frac{\left(\frac{\mathrm{d}}{\mathrm{d} s} Q(s)\right)^{2}}{2 Q(s)}+\frac{9 Q^{3}(s)}{8}+3 Q^{2}(s)+\frac{3 Q(s)}{2}=0 .
$$

Multiplying both sides by $2 Q^{\prime}(s) / Q(s)$ and we have

$$
\frac{\mathrm{d}}{\mathrm{d} s}\left[\frac{\left(Q^{\prime}(s)\right)^{2}}{Q(s)}\right]=\frac{\mathrm{d}}{\mathrm{d} s}\left(-\frac{3}{4} Q^{3}(s)-3 Q^{2}(s)-3 Q(s)\right) .
$$

Integrating with respect to $s$ we have

$$
\left(Q^{\prime}(s)\right)^{2}=-\frac{3}{4} Q^{4}(s)-3 Q^{3}(s)-3 Q^{2}(s)+C_{1} Q(s) .
$$

Letting $\operatorname{Re}(s) \rightarrow-\infty$ we have $Q(s) \rightarrow-2 / 3$ and $Q^{\prime}(s) \rightarrow 0$. Thus $C_{1}=-8 / 9$ and the equation (4.13) is of the form

$$
\left(Q^{\prime}(s)\right)^{2}=-\frac{3}{4} Q(s)\left(Q(s)+\frac{8}{3}\right)\left(Q(s)+\frac{2}{3}\right)^{2} .
$$

This is a separable differential equation with general solutions

$$
Q(s)=-\frac{2}{3} \frac{\mathrm{e}^{2 s}-C_{2}^{2}-2 \mathrm{i} C_{2} \mathrm{e}^{s}}{\mathrm{e}^{2 s}-C_{2}^{2}+\mathrm{i} C_{2} \mathrm{e}^{s}} .
$$

Hence the solution of (3.16) satisfying (2.9) is

$$
F_{0}(\xi)=\frac{4 \xi}{\xi^{2}+2 \xi+4}
$$

\subsubsection{Solving (3.22)}

Make the substitution $Q(s)=F_{0}\left(\mathrm{e}^{s}\right)-2$ then (3.16) transforms into

$$
\frac{\mathrm{d}^{2}}{\mathrm{~d} s^{2}} Q(s)-\frac{\left(\frac{\mathrm{d}}{\mathrm{d} s} Q(s)\right)^{2}}{2 Q(s)}-\frac{3 Q^{3}(s)}{8}-Q^{2}(s)-\frac{Q(s)}{2}=0 .
$$

Multiplying both sides by $2 Q^{\prime}(s) / Q(s)$ and we have

$$
\frac{\mathrm{d}}{\mathrm{d} s}\left[\frac{\left(Q^{\prime}(s)\right)^{2}}{Q(s)}\right]=\frac{\mathrm{d}}{\mathrm{d} s}\left(\frac{1}{4} Q^{3}(s)+Q^{2}(s)+Q(s)\right) .
$$

Integrating with respect to $s$ we have

$$
\left(Q^{\prime}(s)\right)^{2}=\frac{1}{4} Q^{4}(s)+Q^{3}(s)+Q^{2}(s)+C_{1} Q(s) .
$$

Letting $\operatorname{Re}(s) \rightarrow-\infty$ we have $Q(s) \rightarrow-2$ and $Q^{\prime}(s) \rightarrow 0$. Thus $C_{1}=0$ and the equation (4.14) is of the form

$$
\left(Q^{\prime}(s)\right)^{2}=\frac{1}{4} Q^{2}(s)(Q(s)+2)^{2} .
$$

This differential equation has general solutions

$$
Q(s)=-\frac{2 C_{2}}{C_{2}+\mathrm{e}^{s}}
$$

Hence the solution of (3.22) satisfying (2.9) is

$$
F_{0}(\xi)=\frac{2 \xi}{\xi+2} \text {. }
$$




\section{A Appendix}

Recall that the Borel transform of a formal series

$$
\tilde{f}(w)=\sum_{n=0}^{\infty} a_{n} w^{-r-n}, \quad \operatorname{Re}(r)>0,
$$

where the series $\sum_{n=0}^{\infty} a_{n} x^{n}$ has a positive radius of convergence, is defined to be the formal power series

$$
(\mathcal{B} \tilde{f})(p):=\sum_{n=0}^{\infty} \frac{a_{n} p^{n+r-1}}{\Gamma(n+r)} .
$$

Lemma A.1. Assume that we have two formal series $\tilde{f}$ and $\tilde{g}$,

$$
\begin{aligned}
\tilde{f}(w) & =\sum_{n=0}^{\infty} a_{n} w^{-r-n}, & \operatorname{Re}(r)>0, \\
\tilde{g}(w) & =\sum_{n=0}^{\infty} b_{n} w^{-r-s}, & \operatorname{Re}(s)>0,
\end{aligned}
$$

where both series $\sum_{n=0}^{\infty} a_{n} x^{n}$ and $\sum_{n=0}^{\infty} b_{n} x^{n}$ have positive radii of convergence. Then

$$
\mathcal{B}(\tilde{f} \tilde{g})(p)=(\mathcal{B} \tilde{f} * \mathcal{B} \tilde{g})(p)=p^{r+s-1} \sum_{n=0}^{\infty}\left(\sum_{k=0}^{n} a_{k} b_{n-k}\right) \frac{p^{n}}{\Gamma(n+r+s)},
$$

where

$$
(\mathcal{B} \tilde{f} * \mathcal{B} \tilde{g})(p):=\int_{0}^{p}(\mathcal{B} \tilde{f})(t)(\mathcal{B} \tilde{g})(p-t) \mathrm{d} t .
$$

Recall that the Laplace transform $\mathcal{L}_{\phi}$ is defined as the following

$$
f \longmapsto \int_{0}^{\infty \mathrm{e}^{\mathrm{i} \phi}} f(p) \mathrm{e}^{-x p} \mathrm{~d} p
$$

where $\phi \in \mathbb{R}$.

Lemma A.2. Assume that the function $f$ is integrable over the ray $\mathrm{e}^{\mathrm{i} \phi} \mathbb{R}^{+}$, namely

$$
\int_{0}^{\infty \mathrm{e}^{\mathrm{i} \phi}}|f(p)||\mathrm{d} p|<\infty .
$$

Then for $\operatorname{Re}\left(w \mathrm{e}^{\mathrm{i} \phi}\right)>0$,

$$
\mathcal{L}_{\phi}(1 * f)(w)=\frac{1}{w} \mathcal{L}_{\phi}(f)(w)
$$

\section{Acknowledgements}

I am very grateful for the advice and help of Professors Ovidiu Costin and Rodica Costin when I worked on this problem. I also greatly appreciate the referees whose comments helped me improve my paper significantly. 


\section{References}

[1] Boutroux P., Recherches sur les transcendantes de M. Painlevé et l'étude asymptotique des équations différentielles du second ordre, Ann. Sci. École Norm. 30 (1913), 255-375.

[2] Clarkson P.A., Painlevé equations - nonlinear special functions, in Orthogonal Polynomials and Special Functions, Lecture Notes in Math., Vol. 1883, Springer, Berlin, 2006, 331-411.

[3] Costin O., Exponential asymptotics, transseries, and generalized Borel summation for analytic, nonlinear, rank-one systems of ordinary differential equations, Int. Math. Res. Not. 1995 (1995), 377-417, math.CA/0608414.

[4] Costin O., On Borel summation and Stokes phenomena for rank-1 nonlinear systems of ordinary differential equations, Duke Math. J. 93 (1998), 289-344, math.CA/0608408.

[5] Costin O., Topological construction of transseries and introduction to generalized Borel summability, in Analyzable Functions and Applications, Contemp. Math., Vol. 373, Amer. Math. Soc., Providence, RI, 2005, 137-175, math.CA/0608309.

[6] Costin O., Costin R.D., On the formation of singularities of solutions of nonlinear differential systems in antistokes directions, Invent. Math. 145 (2001), 425-485, math.CA/0202234.

[7] Costin O., Costin R.D., Asymptotic properties of a family of solutions of the Painlevé equation $\mathrm{P}_{\mathrm{VI}}$, Int. Math. Res. Not. 2002 (2002), 1167-1182, math.CA/0202235.

[8] Costin O., Costin R.D., Huang M., Tronquée solutions of the Painlevé equation PI, Constr. Approx. 41 (2015), 467-494, arXiv:1310.5330.

[9] Costin O., Huang M., Tanveer S., Proof of the Dubrovin conjecture and analysis of the tritronquée solutions of $\mathrm{P}_{\mathrm{I}}$, Duke Math. J. 163 (2014), 665-704, arXiv:1209.1009.

[10] Dubrovin B., Hamiltonian PDEs: deformations, integrability, solutions, J. Phys. A: Math. Theor. 43 (2010), 434002, 20 pages.

[11] Dubrovin B., Grava T., Klein C., On universality of critical behavior in the focusing nonlinear Schrödinger equation, elliptic umbilic catastrophe and the tritronquée solution to the Painlevé-I equation, J. Nonlinear Sci. 19 (2009), 57-94, arXiv:0704.0501.

[12] Dubrovin B., Kapaev A., On an isomonodromy deformation equation without the Painlevé property, Russ. J. Math. Phys. 21 (2014), 9-35, arXiv:1301.7211.

[13] Fasondini M., Fornberg B., Weideman J.A.C., A computational exploration of the McCoy-Tracy-Wu solutions of the third Painlevé equation, Phys. D 363 (2018), 18-43.

[14] Fokas A.S., Its A.R., Kapaev A.A., Novokshenov V.Yu., Painlevé transcendents. The Riemann-Hilbert approach, Mathematical Surveys and Monographs, Vol. 128, Amer. Math. Soc., Providence, RI, 2006.

[15] Grava T., Kapaev A., Klein C., On the tritronquée solutions of P $\mathrm{I}_{\mathrm{I}}^{2}$, Constr. Approx. 41 (2015), 425-466, arXiv:1306.6161.

[16] Huang M., Xu S.-X., Zhang L., Location of poles for the Hastings-McLeod solution to the second Painlevé equation, Constr. Approx. 43 (2016), 463-494, arXiv:1410.3338.

[17] Its A.R., Kapaev A.A., Quasi-linear Stokes phenomenon for the second Painlevé transcendent, Nonlinearity 16 (2003), 363-386, nlin.SI/0108010.

[18] Joshi N., Kitaev A.V., On Boutroux's tritronquée solutions of the first Painlevé equation, Stud. Appl. Math. 107 (2001), 253-291.

[19] Joshi N., Mazzocco M., Existence and uniqueness of tri-tronquée solutions of the second Painlevé hierarchy, Nonlinearity 16 (2003), 427-439, math.CA/0212117.

[20] Kapaev A.A., Asymptotic behavior of the solutions of the Painlevé equation of the first kind, Differential Equations 24 (1988), 1107-1115.

[21] Kapaev A.A., Global asymptotics of the fourth Painlevé transcendent, Steklov Math. Inst. and IUPUI Preprint \# 96-5, 1996.

[22] Kapaev A.A., Quasi-linear stokes phenomenon for the Painlevé first equation, J. Phys. A:Math. Gen. 37 (2004), 11149-11167, nlin.SI/0404026.

[23] Kapaev A.A., Quasi-linear Stokes phenomenon for the Hastings-McLeod solution of the second Painlevé equation, nlin.SI/0411009.

[24] Kapaev A.A., Kitaev A.V., Connection formulae for the first Painlevé transcendent in the complex domain, Lett. Math. Phys. 27 (1993), 243-252. 
[25] Kitaev A.V., Vartanian A.H., Connection formulae for asymptotics of solutions of the degenerate third Painlevé equation. I, Inverse Problems 20 (2004), 1165-1206, math.CA/0312075.

[26] Kitaev A.V., Vartanian A.H., Connection formulae for asymptotics of solutions of the degenerate third Painlevé equation: II, Inverse Problems 26 (2010), 105010, 58 pages, arXiv:1005.2677.

[27] Lin Y., Dai D., Tibboel P., Existence and uniqueness of tronquée solutions of the third and fourth Painlevé equations, Nonlinearity 27 (2014), 171-186, arXiv:1306.1317.

[28] Masoero D., Poles of intégrale tritronquée and anharmonic oscillators. Asymptotic localization from WKB analysis, Nonlinearity 23 (2010), 2501-2507, arXiv:1002.1042.

[29] McCoy B.M., Tracy C.A., Wu T.T., Painlevé functions of the third kind, J. Math. Phys. 18 (1977), 10581092.

[30] Milne A.E., Clarkson P.A., Bassom A.P., Bäcklund transformations and solution hierarchies for the third Painlevé equation, Stud. Appl. Math. 98 (1997), 139-194.

[31] Novokshenov V.Yu., Distributions of poles to Painlevé transcendents via Padé approximations, Constr. Approx. 39 (2014), 85-99. 